QUARTERLY OF APPLIED MATHEMATICS

VOLUME LXVIII, NUMBER 2

JUNE 2010, PAGES 229-253

S 0033-569X(10)01135-0

Article electronically published on March 10, 2010

\title{
HIGH ORDER MULTI-SCALE WALL-LAWS, PART I: THE PERIODIC CASE
}

\author{
BY \\ DIDIER BRESCH (LAMA, UMR 5127 CNRS, Université de Savoie, 73217 Le Bourget du Lac cedex, \\ France) \\ AND \\ VUK MILISIC (LJK-IMAG, UMR 5523 CNRS, 51 rue des Mathématiques, B.P.53, 38041 Grenoble \\ cedex 9, France)
}

\begin{abstract}
In this work we present new wall-laws boundary conditions including microscopic oscillations. We consider a Newtonian flow in domains with periodic rough boundaries that we simplify considering a Laplace operator with periodic inflow and outflow boundary conditions. Following the previous approaches, see [A. Mikelić, W. Jäger, J. Diff. Eqs, 170, 96-122, (2001)] and [Y. Achdou et al, J. Comput. Phys., 147, 1, 187-218, (1998)], we construct high order boundary layer approximations and rigorously justify their rates of convergence with respect to $\epsilon$ (the roughness' thickness). We establish mathematically a poor convergence rate for averaged second order wall-laws as it was illustrated numerically for instance in [Y. Achdou, et al]. In comparison, we establish exponential error estimates in the case of an explicit multi-scale ansatz. This motivates our study to derive implicit first order multi-scale wall-laws and to show that their rate of convergence is at least of order $\epsilon^{\frac{3}{2}}$. We provide a numerical assessment of the claims as well as a counterexample that makes evident the impossibility of an averaged second order wall-law. Our paper may be seen as the first one to derive efficient high order wall-laws boundary conditions.
\end{abstract}

Received February 20, 2008.

2000 Mathematics Subject Classification. Primary 76D05, 35B27, 76Mxx, 65Mxx.

Key words and phrases. Wall-laws, rough boundary, Laplace equation, multi-scale modelling, boundary layers, finite element methods, error estimates.

The first author was partially supported by the project "Études mathématiques de paramétrisations en océanographie" that is part of the "ACI jeunes chercheurs 2004" framework of the French Research Ministry and by a Rhône Alpes project "Equations de type Saint-Venant avec viscosité pour des problèmes environnementaux".

The second author was partially supported by a contract with Cardiatis ${ }^{\circledR}$, a company providing metallic multi-layer stents for cerebral and aortic aneurysms. This research has been partly funded by the RhôneAlpes Institute of Complex Systems IXXI, http://www.ixxi.fr. The authors would like to thank E. Bonnetier for fruitful discussions and helpful proofreading.

E-mail address: didier.bresch@univ-savoie.fr

E-mail address: vuk.milisic@imag.fr

(C)2010 Brown University Reverts to public domain 28 years from publication 


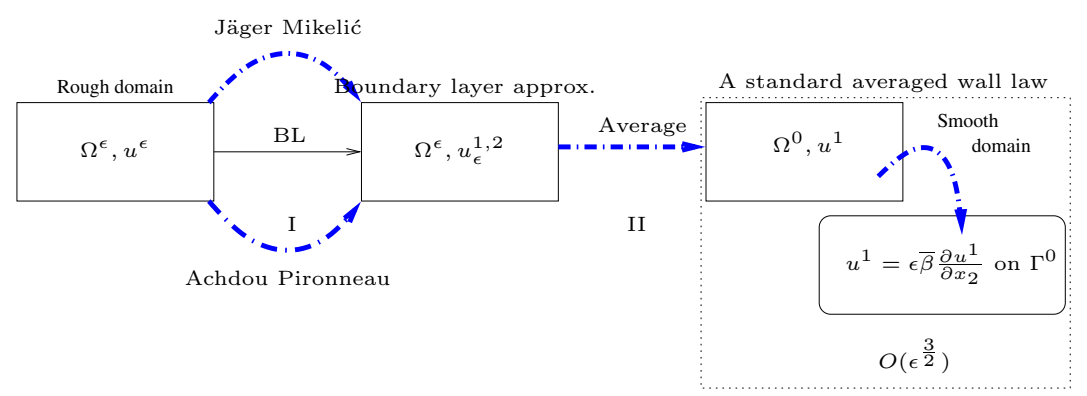

FIG. 1. The standard approach: from the exact solution to an averaged wall-law

1. Introduction. The main goal of wall-laws is to remove the stiff part from boundary layers, replacing the classical no-slip boundary condition by a more sophisticated relation between the variables and their derivatives. They are extensively used in numerical simulations to eliminate regions of strong gradients or regions of complex geometry (rough boundaries) from the domain of computation. Depending on the field of applications (porous media, fluid mechanics, heat transfer, electromagnetism), wall-laws may be called Beavers-Joseph, Saffman-Joseph, Navier, Fourier, or Leontovitch type laws.

High order effective macroscopic boundary conditions may also be proposed if we choose a higher degree ansatz; see [10 for applications in microfluidics. In a similar perspective but in the context of fluid mechanics, numerical simulations have shown that second order macroscopic wall-laws provide the same order of approximation as the first order approximation. Recently a generalized wall-law formulation has been obtained for curved rough boundaries [26, 28] and for random roughness [5]. Note that such generalizations are important from a practical point of view when dealing with e.g. coastal effects in geophysical flows. From a mathematical point of view, wall-laws are also interesting. In the proof of convergence to the Euler equations, the 2D Navier-Stokes system is complemented with wall-laws of the NAVIER type [9]. Recently several papers have analyzed in various settings the properties of such boundary conditions; see [17, [25], 16], 8], [18, 2].

In this paper, we focus on fluid flows. Starting from the Stokes system, we simplify the problem by studying the axial velocity through the resolution of a specific Poisson problem with periodic inlet and outlet boundary conditions. Our scope is to justify mathematically higher order macroscopic wall-laws and to explain why in their averaged form they do not provide better results than the first order laws. We shall explain how to get better estimates including some coefficients depending on the microscopic variables: this leads to new oscillating wall-laws.

The basic scheme to establish standard averaged wall-laws is the following (see Fig. (1). First we use an ansatz for the velocity and the pressure, which will give, after an adequate extension, a main order term completed with some boundary layer correctors defined on the whole rough domain (Fig. 1, step I). This is possible due to the boundary layer theory, which can be seen as a particular case of a general homogenization process. In a 


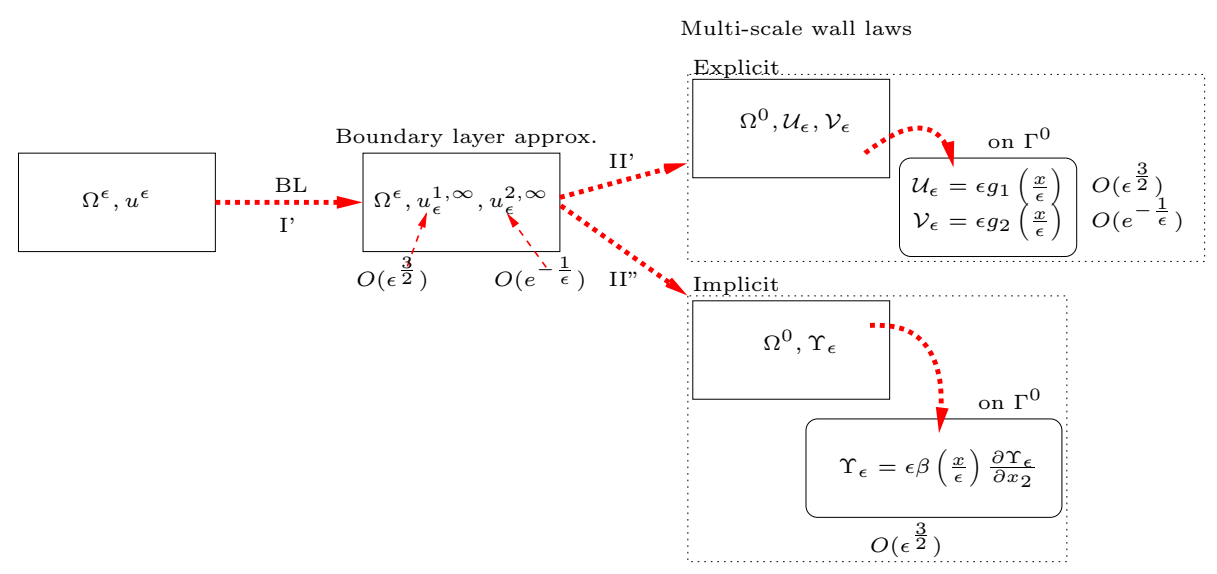

Fig. 2. The new approach: from the exact solution to multi-scale wall-laws

second step, a specific average is performed on this approximation and a new boundary condition of mixed type is recovered on a smooth fictitious interface strictly contained in the domain (Fig. 1, step II). As one sees on the figure the only difference between Achdou's and Jäger's approaches is situated in the boundary layer's construction. It is an easy task to show that they are in fact a specific lift, one of the other 7 .

The main result in our paper is the derivation of a high order boundary layer approximation that satisfies the homogeneous Dirichlet boundary condition on the rough wall and that leads to new wall-laws with microscopic effects; see Fig. 2, The ansatz is expanded up to the second order in $\epsilon$, and an exponential convergence in the interior domain is obtained using it (Fig. 2, step $\mathrm{I}^{\prime}$ ). Despite this high order convergence, the corresponding second order averaged wall-law does not conserve this property and is only first order accurate. The estimates show the importance of microscopic oscillations. We then derive new wall-laws that do converge exponentially on the smooth domain. They have the form of explicit non-homogeneous Dirichlet boundary conditions and they depend on the zeroth order Poiseuille flow as well as on the microscopic oscillations on the fictitious interface (Fig. 2, step $\mathrm{II}^{\prime}$ ).

At this stage, we go one step further and derive an implicit multi-scale first order wall-law. We obtain a SAFFMAN-JoSEPH's like law that now contains a coefficient that includes the microscopic oscillations. We rigorously derive a rate of convergence in $\epsilon^{\frac{3}{2}}$, thanks to the steps introduced in the previous sections (Fig. 2, step II").

We underline that this work is a necessary building block when studying wall-laws for the stationary Navier-Stokes equations: asymptotic expansion of the quadratic nonlinearity transfers a cascade of contributions to the microscopic cell problems, as already noticed in [1. The first order cell problem is homogeneous and the second-order cell problem involves the non-linearity of the first order approximation. Until now, averaged wall-laws were only first order accurate, so that they do not take into account second order non-linear effects. 


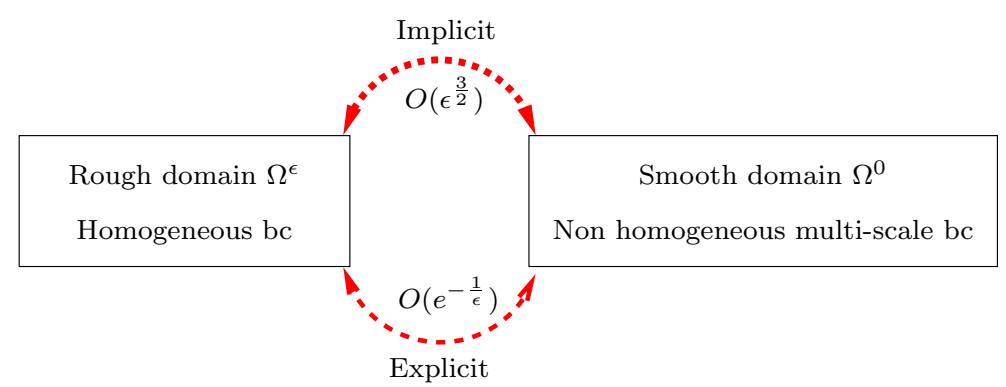

FIG. 3. One of the main points of this article: switching perturbations from geometry to boundary data. (BC stands for boundary conditions.)

Although the authors are conscious that wall-laws aim to reduce complexity by "homogenizing" microscopic scales into averaged macroscopic quantities, we believe that implicit multi-scale wall-laws are indeed of interest for the following reasons. At first, one should compute the microscopic cell problem anyway to compute the averaged coefficient $\bar{\beta}$ (see below) used in the wall-law, so using the trace of $\beta$ on the fictitious interface rather than its mean value makes sense. Then, as shown in the numerical tests below, the microscopic oscillations induced by the multi-scale Robin condition improve the wall-law approximation. In a wider context that does not concern only fluid flows, the main concept that this work emphasizes is the following: we have shown that it is possible to replace a geometrical roughness and "smooth" boundary conditions (in the unperturbed sense, as for instance homogeneous Dirichlet ones) by a smooth domain but with multi-scale perturbed boundary conditions (see Fig. 31). Depending on the kind of boundary perturbation, we get different orders of precision in this process. For complex multi-scale 3D problems, we still expect some numerical gain when performing this switch, especially if one uses some increased multi-scale finite element bases (see [13] and the references therein).

To show the practical importance of the above results, in Section 6 , we perform numerical tests on a $2 \mathrm{D}$ case. For various values of $\epsilon$, we first compute the rough solution $u_{\Delta}^{\epsilon}$ on the whole domain $\Omega^{\epsilon}$. Then we compute the wall-law solutions defined only on the interior smooth domain $\Omega^{0}$. We perform these tests in the periodic case. We recover exactly theoretical claims: numerical error estimates confirm that averaged wall-laws do not differ at first and second orders. We prove that our new implicit multi-scale wall-law provides better results than classical averaged laws. However, the fully explicit approximations still show higher order convergence rates with respect to $\epsilon$.

2. The simplified problem: from Navier-Stokes to the Laplace equation. In

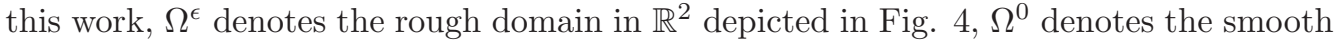
one, and $\Lambda^{\epsilon}:=\Omega^{\epsilon} \backslash \Omega^{0}$ the complementary rough subdomain. $\Gamma^{\epsilon}$ is the rough boundary and $\Gamma^{0}$ (resp. $\Gamma^{1}$ ) the lower (resp. upper) smooth one (see Fig. 4 ).

Hyротнeses 2.1. The rough boundary $\Gamma^{\epsilon}$ is described as a periodic repetition at the microscopic scale of a single boundary cell $P^{0}$. The latter can be parameterized as the 
graph of a Lipshitz function $f:[0,2 \pi[\rightarrow[-1: 0[$ such that

$$
P^{0}=\left\{y \in[0,2 \pi] \times\left[-1: 0\left[\text { s.t. } y_{2}=f\left(y_{1}\right)\right\} .\right.\right.
$$

Moreover, we suppose that $f$ is negative definite, i.e. that there exists a positive constant $\delta$ such that $f\left(y_{1}\right)<\delta$ for all $y_{1} \in[0,2 \pi]$. Then the macroscopic boundary $\Gamma^{\epsilon}$ is parametrized as

$$
\Gamma^{\epsilon}=\left\{x \in \mathbb{R}^{2} \text { s.t. } x_{2}=\epsilon f\left(\frac{x}{\epsilon}\right)\right\} .
$$

We assume that the ratio between $L$ (the width of $\Omega^{0}$ ) and $2 \pi \epsilon$ (the width of the periodic cell) is always an integer called $N$. We consider a simplified setting that avoids the
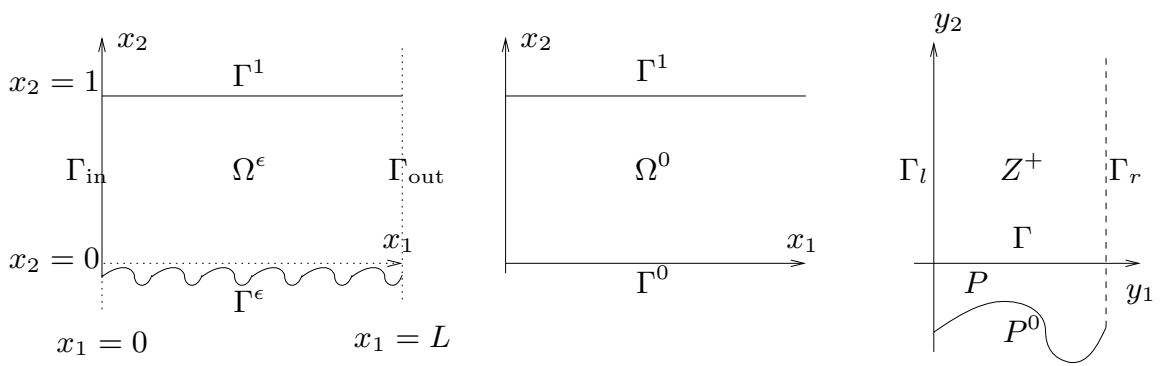

FIG. 4. Rough, smooth and cell domains

theoretical difficulties and the non-linear complications of the full Navier-Stokes equations. Starting from the Stokes system, we consider a Poisson problem for the axial component of the velocity. The pressure gradient is assumed to reduce to a constant right-hand side $C$. We consider only periodic inflow and outflow boundary conditions. The simplified problem reads : find $u^{\epsilon}$ such that

$$
\left\{\begin{array}{l}
-\Delta u^{\epsilon}=C, \quad \text { for } x \in \Omega^{\epsilon}, \\
u^{\epsilon}=0, \quad x \in \Gamma^{\epsilon} \cup \Gamma^{1}, \\
u^{\epsilon} \text { is } x_{1} \text { periodic. }
\end{array}\right.
$$

We underline that the results below can be directly extended to rough domains with smooth holes and to the Stokes system in the case of a simple sheared flow.

In what follows, functions that do depend on $y=x / \epsilon$ should be indexed by an $\epsilon$ (e.g. $\left.\mathcal{U}_{\epsilon}=\mathcal{U}_{\epsilon}(x, x / \epsilon)\right)$.

\section{The full boundary layers correctors.}

3.1. A zeroth order approximation. When $\epsilon=0$, the rough domain $\Omega^{\epsilon}$ reduces to $\Omega^{0}$, which is smooth. The solution of system (2) in this limit is known and explicit: it is the Poiseuille profile :

$$
\tilde{u}^{0}(x)=\frac{C}{2}\left(1-x_{2}\right) x_{2}, \quad \forall x \in \Omega^{0} ;
$$

the latter term should be our zeroth order approximation when performing an asymptotic expansion w.r.t. $\epsilon$ for $\epsilon>0$. Although the natural extension of $\tilde{u}^{0}$ is itself on the rough part $\Lambda^{\epsilon}$, what follows is more general and concerns the case for instance where $\Delta u^{\epsilon}$ is no 
longer a constant. In the literature the main difference between [1] and [19] is the way of extending this zeroth order approximation in order to define it on the whole domain $\Omega^{\epsilon}$. A possible choice (see [1]) is to use the Taylor expansion of $\tilde{u}^{0}$ near $x_{2}=0$; this leads to defining the zeroth order expansion as a $C^{1}\left(\Omega^{\epsilon}\right)$ function that reads

$$
u_{1}^{0}(x)= \begin{cases}\tilde{u}^{0}(x), & \text { if } x \in \Omega^{0}, \\ \frac{\partial \tilde{u}^{0}}{\partial x_{2}}\left(x_{1}, 0\right) x_{2}, & \text { if } x \in \Lambda^{\epsilon} .\end{cases}
$$

Notice that this particular choice does not satisfy the homogeneous Dirichlet boundary condition on $\Gamma^{\epsilon}$. Next we estimate the zeroth order error w.r.t. the exact solution.

Proposition 1. If $\Omega^{\epsilon}$ is an open connected piecewise smooth domain, the solution $u^{\epsilon}$ exists in $H^{1}\left(\Omega^{\epsilon}\right)$ and is unique. Moreover we have

$$
\left\|u^{\epsilon}-u_{1}^{0}\right\|_{H^{1}\left(\Omega^{\epsilon}\right)} \leq c_{1} \sqrt{\epsilon}, \quad\left\|u^{\epsilon}-u_{1}^{0}\right\|_{L^{2}\left(\Omega^{0}\right)} \leq c_{2} \epsilon,
$$

where the constants $c_{1}$ and $c_{2}$ are independent of $\epsilon$.

Proof. The proof is based on standard a priori estimates and a duality argument. The existence and uniqueness of $u^{\epsilon}$ are standard and left to the reader. We focus on the error estimates. Namely, $r^{0}:=u^{\epsilon}-u^{0}$ satisfies

$$
\left\{\begin{array}{l}
-\Delta r^{0}=C \chi_{\left[\Lambda^{\epsilon}\right]} \text { in } \Omega^{\epsilon}, \\
r^{0}=0 \text { on } \Gamma^{1}, \\
r^{0}=-\frac{\partial \tilde{u}^{0}}{\partial x_{2}}\left(x_{1}, 0\right) x_{2} \text { on } \Gamma^{\epsilon}, \\
r^{0} \text { is } x_{1} \text {-periodic on } \Gamma_{\text {in }} \cup \Gamma_{\text {out }} .
\end{array}\right.
$$

Here and for the rest of the work, $\chi_{[\cdot]}$ represents the characteristic function of the set between brackets. Above, one remarks that a part of the error comes from the source term localized in $\Lambda^{\epsilon}$, and another part comes from the non-homogeneous boundary term on $\Gamma^{\epsilon}$. Setting the lift

$$
s=-\frac{\partial \tilde{u}^{0}}{\partial x_{2}} x_{2} \chi_{[\Lambda \epsilon]} \text { and } z:=r^{0}-s,
$$

the weak formulation reads:

$$
(\nabla z, \nabla v)_{\Omega^{\epsilon}}=(C, v)_{\Lambda^{\epsilon}}-(C, v)_{\Gamma^{0}}, \quad v \in H_{0}^{1}\left(\Omega^{\epsilon}\right),
$$

where the last term in the right-hand side comes when applying the Laplace operator $\Delta$ on $s$. Thanks to Poincaré-like estimates we have the following properties of the $L^{2}$ norm and the $H^{1}$ semi-norm on $\Lambda^{\epsilon}$ :

$$
\begin{aligned}
\left|(C, v)_{\Lambda^{\epsilon}}-(C, v)_{\Gamma^{0}}\right| & \leq c_{3} \sqrt{\epsilon}\left(\int_{\Lambda^{\epsilon}} v^{2}\right)^{\frac{1}{2}}+c_{4}\left(\int_{\Gamma^{0}} v^{2}\right)^{\frac{1}{2}} \\
& \leq c_{5}(\epsilon+\sqrt{\epsilon})\left(\int_{\Lambda^{\epsilon}}|\nabla v|^{2}\right)^{\frac{1}{2}} \leq 2 c_{5} \sqrt{\epsilon}\|v\|_{H^{1}\left(\Omega^{\epsilon}\right)}
\end{aligned}
$$


This leads to the $H^{1}\left(\Omega^{\epsilon}\right)$ estimate. For the $L^{2}$ norm, we use the concept of a very weak solution [27. Namely, one solves the dual problem: for a given $\varphi \in L^{2}\left(\Omega^{0}\right), \varphi$ being $x_{1}$-periodic on $\Gamma_{\text {in }} \cup \Gamma_{\text {out }}$ find $v \in H^{2}\left(\Omega^{0}\right)$ such that

$$
\left\{\begin{array}{l}
-\Delta v=\varphi, \quad \forall x \in \Omega^{0} \\
v=0, \quad \forall x \in \Gamma^{0} \cup \Gamma^{1} \\
v \text { is } x_{1} \text {-periodic on } \Gamma_{\text {in }} \cup \Gamma_{\text {out }} .
\end{array}\right.
$$

Considering the $L^{2}\left(\Omega^{0}\right)$ scalar product, and using the Green formula

$$
\begin{aligned}
\left(\varphi, r^{0}\right)_{\Omega^{0}} & =-\left(\Delta v, r^{0}\right)_{\Omega^{0}}=\left\langle\frac{\partial r^{0}}{\partial \mathbf{n}}, v\right\rangle_{\partial \Omega^{0}}-\left(\frac{\partial v}{\partial \mathbf{n}}, r^{0}\right)_{L^{2}\left(\partial \Omega^{0}\right)}-\left(v, \Delta r^{0}\right)_{\Omega^{0}} \\
& =\left\langle v, \frac{\partial r^{0}}{\partial \mathbf{n}}\right\rangle_{\Gamma_{\text {in }} \cup \Gamma_{\text {out }}}-\left(\frac{\partial v}{\partial \mathbf{n}}, r^{0}\right)_{\Gamma^{0} \cup \Gamma^{1}},
\end{aligned}
$$

where the brackets refer to the dual product in $\left(H^{-1}, H^{1}\right)\left(\partial \Omega^{0}\right)$, and the remaining products are in $L^{2}$, either on $\Gamma^{0}$ or on $\Omega^{0}$. Then, one computes

$$
\left|\left(\varphi, r^{0}\right)\right| \leq\left|\left(\frac{\partial v}{\partial \mathbf{n}}, r^{0}\right)_{\Gamma^{0}}\right| \leq\left\|\frac{\partial v}{\partial \mathbf{n}}\right\|_{L^{2}\left(\Gamma^{0}\right)}\left\|r^{0}\right\|_{L^{2}\left(\Gamma^{0}\right)} \leq c_{6}\|\varphi\|_{L^{2}\left(\Omega^{0}\right)}\left\|r^{0}\right\|_{L^{2}\left(\Gamma^{0}\right)}
$$

The last estimate is classical and can be obtained via a regularisation of the Lipshitz domain and the Rellich estimates ([27], pp. 261-263). Thanks to Poincaré estimates, one writes

$$
\left\|r^{0}\right\|_{L^{2}\left(\Gamma^{0}\right)} \leq c_{7} \sqrt{\epsilon}\left\|r^{0}\right\|_{H^{1}\left(\Lambda^{\epsilon}\right)} \leq c_{8} \sqrt{\epsilon}\left\|r^{0}\right\|_{H^{1}\left(\Omega^{\epsilon}\right)},
$$

which ends the proof by taking the sup over all $\varphi$ in $L^{2}\left(\Omega^{0}\right)$.

3.2. A first order correction. The zeroth order correction contains two distinct sources of errors: one part is due to the order of the extension in $\Lambda^{\epsilon}$ and another part comes from a non-homogeneous remainder on $\Gamma^{\epsilon}$. In what follows we show that a first order extension $u_{1}^{0}$ can be corrected by series of terms that make the full boundary layer approximation vanish on $\Gamma^{\epsilon}$.

The microscopic cell problem. In order to correct $u_{1}^{0}$ on $\Gamma^{\epsilon}$, one starts by solving a microscopic cell problem that reads: find $\beta$ s.t.

$$
\left\{\begin{array}{l}
-\Delta \beta=0, \text { in } Z^{+} \cup P, \\
\beta=-y_{2}, \text { on } P^{0}, \\
\beta \text { is } y_{1} \text {-periodic } .
\end{array}\right.
$$

We define the microscopic average along the fictitious interface $\Gamma$ :

$$
\bar{\beta}=\frac{1}{2 \pi} \int_{0}^{2 \pi} \beta\left(y_{1}, 0\right) d y_{1} .
$$

The existence and uniqueness of $\beta$ and various properties as the convergence when $y_{2}$ tends to inflinity towards $\bar{\beta}$ are described in Appendix $\mathrm{A}$

The corresponding macroscopic full boundary layer corrector should contain at this stage

$$
u_{1}^{0}+\epsilon \frac{\partial u_{1}^{0}}{\partial x_{2}}\left(x_{1}, 0\right)\left(\beta\left(\frac{x}{\epsilon}\right)-\bar{\beta}\right),
$$


where we subtract $\bar{\beta}$ in order to cancel $\beta$ 's errors on $\Gamma^{1}$. In turn, we expect to cancel the contribution of $\bar{\beta}$ near the rough boundary but keep it close to $\Gamma^{1}$. For this sake, we solve the "counterflow" problem: find $d$ s.t.

$$
\left\{\begin{array}{l}
-\Delta d=0, \quad \text { in } \Omega^{0}, \\
d=1 \text { on } \Gamma^{0}, d=0 \text { on } \Gamma^{1}, \\
d \text { is } x_{1} \text {-periodic on } \Gamma_{\text {in }} \cup \Gamma_{\text {out }},
\end{array}\right.
$$

where the solution is explicit and reads $d=\left(1-x_{2}\right)$. Moreover, it can be extended to the whole domain $\Omega^{\epsilon}$. The complete first order approximation now reads:

$$
\begin{aligned}
u_{\epsilon}^{1,2} & :=u_{1}^{0}+\epsilon \frac{\partial u_{1}^{0}}{\partial x_{2}}\left(x_{1}, 0\right)(\beta-\bar{\beta})+\epsilon \frac{\partial u_{1}^{0}}{\partial x_{2}}\left(x_{1}, 0\right) \bar{\beta}\left(1-x_{2}\right), \quad \forall x \in \Omega^{\epsilon} \\
& =u_{1}^{0}+\epsilon \frac{\partial u_{1}^{0}}{\partial x_{2}}\left(x_{1}, 0\right)\left(\beta-\bar{\beta} x_{2}\right) .
\end{aligned}
$$

The first index of $u_{\epsilon}^{1,2}$ corresponds to the extension order of $\tilde{u}^{0}$ in $\Lambda^{\epsilon}$, while the second index is the order of the error on $\Gamma^{\epsilon}$. Indeed, if we consider the trace of $u_{\epsilon}^{1,2}$ on $\Gamma^{\epsilon}$, we have a second order error

$$
\left.u_{\epsilon}^{1,2}\right|_{\Gamma^{\epsilon}}=\epsilon^{2}\left(\frac{\partial u_{1}^{0}}{\partial x_{2}} \bar{\beta}\right) \frac{x_{2}}{\epsilon}=\epsilon^{2}\left(\frac{\partial u_{1}^{0}}{\partial x_{2}} \bar{\beta}\right) y_{2} .
$$

Again, this error is linear and should be corrected by the micro boundary layer $\beta$. A similar macroscopic boundary layer correction process should be performed at any order, leading to

$$
\begin{aligned}
u_{\epsilon}^{1, \infty}= & u_{1}^{0}+\epsilon \frac{\partial u_{1}^{0}}{\partial x_{2}}\left(x_{1}, 0\right)\left[\left(\beta\left(\frac{x}{\epsilon}\right)-\bar{\beta} x_{2}\right)+\epsilon \bar{\beta}\left(\beta\left(\frac{x}{\epsilon}\right)-\bar{\beta} x_{2}\right)\right. \\
& \left.-\epsilon^{2} \bar{\beta}^{2}\left(\beta\left(\frac{x}{\epsilon}\right)-\bar{\beta} x_{2}\right)+\ldots\right] \\
= & u_{1}^{0}+\frac{\epsilon}{1+\epsilon \bar{\beta}} \frac{\partial u_{1}^{0}}{\partial x_{2}}\left(x_{1}, 0\right)\left(\beta\left(\frac{x}{\epsilon}\right)-\bar{\beta} x_{2}\right) .
\end{aligned}
$$

This approximation satisfies a homogeneous Dirichlet boundary condition on $\Gamma^{\epsilon}$ and solves

$$
\left\{\begin{array}{l}
-\Delta u_{\epsilon}^{1, \infty}=C \chi_{\left[\Lambda^{\epsilon}\right]} \text { in } \Omega^{\epsilon} \\
u_{\epsilon}^{1, \infty}=0 \text { on } \Gamma^{\epsilon} \\
u_{\epsilon}^{1, \infty}=\frac{\epsilon}{1+\epsilon \bar{\beta}} \frac{\partial u_{1}^{0}}{\partial x_{2}}\left(\beta\left(\frac{x_{1}}{\epsilon}, 0\right)-\bar{\beta}\right) \text { on } \Gamma^{1}, \\
u_{\epsilon}^{1, \infty} \text { is } x_{1} \text {-periodic on } \Gamma_{\text {in }} \cup \Gamma_{\text {out }} .
\end{array}\right.
$$

If we consider the corresponding approximation error, we obtain

Proposition 2. Under Hypotheses 2.1, the error of the first order approximation satisfies

$$
\left\|u^{\epsilon}-u_{\epsilon}^{1, \infty}\right\|_{H^{1}\left(\Omega^{\epsilon}\right)} \leq c_{8} \epsilon, \quad\left\|u^{\epsilon}-u_{\epsilon}^{1, \infty}\right\|_{L^{2}\left(\Omega^{0}\right)} \leq c_{9} \epsilon^{\frac{3}{2}}
$$

where the constants $c_{8}, c_{9}$ are independent of $\epsilon$. 
The proof follows the same lines as in Proposition 1 except that the significant source of errors is the right-hand side of the first equation in (7), while an exponentially small microscopic perturbation lies on $\Gamma^{1}$; on the contrary there are no errors on $\Gamma^{\epsilon}$, because $u_{\epsilon}^{1, \infty}=0$ there.

3.3. Second order approximation. Instead of extending only linearly the Poiseuille profile it is obvious that a quadratic term is missing to complete the approximation. In the following $u_{2}^{0}$ denotes the second order extension of $\tilde{u}^{0}$ in $\Lambda^{\epsilon}$ :

$$
u_{2}^{0}:=\left\{\begin{array}{l}
\tilde{u}^{0}, \quad x \in \Omega^{0} \\
\frac{\partial \tilde{u}^{0}}{\partial x_{2}}\left(x_{1}, 0\right) x_{2}+\frac{\partial^{2} \tilde{u}^{0}}{\partial x_{2}^{2}}\left(x_{1}, 0\right) \frac{x_{2}^{2}}{2}, \quad x \in \chi_{\left[\Lambda^{\epsilon}\right]}
\end{array}\right\}=\frac{C}{2}\left(1-x_{2}\right) x_{2}, \quad \forall x \in \Omega^{\epsilon} .
$$

The second order error on $\Gamma^{\epsilon}$ is corrected thanks to a new cell problem: find $\gamma \in D^{1,2}$ solving

$$
\left\{\begin{array}{l}
-\Delta \gamma=0, \quad \text { in } Z^{+} \cup P \\
\gamma=-y_{2}^{2}, \quad \text { on } P^{0} \\
\gamma \text { is periodic in } y_{1}
\end{array}\right.
$$

The proof of the following proposition is left to Appendix A.2.

Proposition 3. Under Hypotheses 2.1 there exists a unique solution $\gamma$ of (8) in $D^{1,2}\left(Z^{+} \cup P\right)$. Moreover it admits a power series of Fourier modes in $Z^{+}$and $\gamma \in[-1,0]$ if $P \subset[0,2 \pi] \times[-1,0]$.

The horizontal average is denoted by $\bar{\gamma}$. The same multi-scale process leads us to write the full boundary layer approximation as

$$
u_{\epsilon}^{2,3}=u_{2}^{0}+\frac{\epsilon}{1+\epsilon \bar{\beta}} \frac{\partial u_{2}^{0}}{\partial x_{2}}\left(x_{1}, 0\right)\left(\beta\left(\frac{x}{\epsilon}\right)-\bar{\beta} x_{2}\right)+\frac{\epsilon^{2}}{2} \frac{\partial^{2} u_{2}^{0}}{\partial x_{2}^{2}}\left(x_{1}, 0\right)\left(\gamma\left(\frac{x}{\epsilon}\right)-\bar{\gamma} x_{2}\right) .
$$

Again a third error remains on $\Gamma^{\epsilon}$ and it is linear w.r.t. to $y_{2}$; thus it should be corrected thanks to the series of first order cell problems as in (6). We set $u_{\epsilon}^{2, \infty}$ to be the second order approximation that satisfies a homogeneous Dirichlet boundary condition on $\Gamma^{\epsilon}$. It reads:

$$
\begin{aligned}
u_{\epsilon}^{2, \infty} & =u_{2}^{0}+\frac{\epsilon}{1+\epsilon \bar{\beta}} \frac{\partial u_{2}^{0}}{\partial x_{2}}\left(x_{1}, 0\right)\left(\beta\left(\frac{x}{\epsilon}\right)-\bar{\beta} x_{2}\right) \\
& +\frac{\epsilon^{2}}{2} \frac{\partial^{2} u_{2}^{0}}{\partial x_{2}^{2}}\left(x_{1}, 0\right)\left[\left(\gamma\left(\frac{x}{\epsilon}\right)-\bar{\gamma} x_{2}\right)+\frac{\epsilon \bar{\gamma}}{1+\epsilon \bar{\beta}}\left(\beta\left(\frac{x}{\epsilon}\right)-\bar{\beta} x_{2}\right)\right] .
\end{aligned}
$$

Our approximation satisfies the following boundary value problem:

$$
\left\{\begin{array}{l}
-\Delta u_{\epsilon}^{2, \infty}=C \text { in } \Omega^{\epsilon} \\
u_{\epsilon}^{2, \infty}=0 \text { on } \Gamma^{\epsilon} \\
u_{\epsilon}^{2, \infty}=g_{\epsilon} \text { on } \Gamma^{1} \\
u_{\epsilon}^{2, \infty} \text { is } x_{1} \text {-periodic on } \Gamma_{\text {in }} \cup \Gamma_{\text {out }},
\end{array}\right.
$$


where $g$ is the contribution of the microscopic correctors on $\Gamma^{1}$ and reads:

$$
\begin{aligned}
g_{\epsilon}= & \frac{\partial u_{2}^{0}}{\partial x_{2}}\left(x_{1}, 0\right)\left(\beta\left(\frac{x_{1}}{\epsilon}, \frac{1}{\epsilon}\right)-\bar{\beta}\right) \\
& +\frac{\epsilon^{2}}{2} \frac{\partial^{2} u_{2}^{0}}{\partial x_{2}^{2}}\left(x_{1}, 0\right)\left[\left(\gamma\left(\frac{x_{1}}{\epsilon}, \frac{1}{\epsilon}\right)-\bar{\gamma}\right)+\frac{\epsilon \bar{\gamma}}{1+\epsilon \bar{\beta}}\left(\beta\left(\frac{x_{1}}{\epsilon}, \frac{1}{\epsilon}\right)-\bar{\beta}\right)\right] .
\end{aligned}
$$

Observe that the only error remains on $\Gamma^{1}$ and as the proposition below claims, it is exponentially small w.r.t. $\epsilon$.

Proposition 4. Under Hypotheses 2.1 the error of the first second order approximation satisfies

$$
\left\|u^{\epsilon}-u_{\epsilon}^{2, \infty}\right\|_{H^{1}\left(\Omega^{\epsilon}\right)} \leq c_{10} e^{-\frac{1}{\epsilon}}, \quad\left\|u^{\epsilon}-u_{\epsilon}^{2, \infty}\right\|_{L^{2}\left(\Omega^{0}\right)} \leq c_{11} \sqrt{\epsilon} e^{-\frac{1}{\epsilon}},
$$

where the constants $c_{6}, c_{7}$ are independent of $\epsilon$.

The proof is identical as in Proposition 1 except that the errors come from $g_{\epsilon}$. There are neither errors on $\Gamma^{\epsilon}$, nor pollution of any source terms inside $\Omega^{\epsilon}$.

\section{Averaged wall-laws.}

4.1. The averaged wall-laws: a new derivation process. At this stage, we rewrite our first and second order approximations, separating slow and fast variables:

$$
\begin{aligned}
u_{\epsilon}^{1, \infty} & =u_{1}^{0}+\frac{\epsilon \bar{\beta}}{1+\epsilon \bar{\beta}} \frac{\partial u_{1}^{0}}{\partial x_{2}}\left(x_{1}, 0\right)\left(1-x_{2}\right)+\frac{\epsilon}{1+\epsilon \bar{\beta}} \frac{\partial u_{1}^{0}}{\partial x_{2}}\left(x_{1}, 0\right)\left(\beta\left(\frac{x}{\epsilon}\right)-\bar{\beta}\right), \\
u_{\epsilon}^{2, \infty} & =u_{2}^{0}+\frac{\epsilon \bar{\beta}}{1+\epsilon \bar{\beta}} \frac{\partial u_{2}^{0}}{\partial x_{2}}\left(x_{1}, 0\right)\left(1-x_{2}\right) \\
& +\frac{\epsilon^{2}}{2} \frac{\partial^{2} u_{2}^{0}}{\partial x_{2}^{2}}\left(x_{1}, 0\right)\left[\bar{\gamma}\left(1-x_{2}\right)+\frac{\epsilon \bar{\gamma} \bar{\beta}}{1+\epsilon \bar{\beta}}\left(1-x_{2}\right)\right] \\
& +\frac{\epsilon}{1+\epsilon \bar{\beta}} \frac{\partial u_{2}^{0}}{\partial x_{2}}\left(x_{1}, 0\right)\left(\beta\left(\frac{x}{\epsilon}\right)-\bar{\beta}\right) \\
& +\frac{\epsilon^{2}}{2} \frac{\partial^{2} u_{2}^{0}}{\partial x_{2}^{2}}\left(x_{1}, 0\right)\left[\left(\gamma\left(\frac{x}{\epsilon}\right)-\bar{\gamma} x_{2}\right)+\frac{\epsilon \bar{\gamma}}{1+\epsilon \bar{\beta}}\left(\beta\left(\frac{x}{\epsilon}\right)-\bar{\beta} x_{2}\right)\right] .
\end{aligned}
$$

We define the average w.r.t. the fast variable in the horizontal direction:

$$
\bar{v}(x)=\frac{1}{2 \pi \epsilon} \int_{0}^{2 \pi \epsilon} v\left(x_{1}+y, x_{2}\right) d y, \quad \forall v \in H^{1}\left(\Omega^{\epsilon}\right) .
$$

Then, one can see easily that for any $x$ in $\Omega^{0}$,

$$
\begin{aligned}
\overline{u_{\epsilon}^{1, \infty}} & =u_{1}^{0}+\frac{\epsilon \bar{\beta}}{1+\epsilon \bar{\beta}} \frac{\partial u_{1}^{0}}{\partial x_{2}}\left(x_{1}, 0\right)\left(1-x_{2}\right)=: u^{1}, \\
\overline{u_{\epsilon}^{2, \infty}} & =u_{2}^{0}+\frac{\epsilon \bar{\beta}}{1+\epsilon \bar{\beta}} \frac{\partial u_{2}^{0}}{\partial x_{2}}\left(x_{1}, 0\right)\left(1-x_{2}\right) \\
& +\frac{\epsilon^{2}}{2} \frac{\partial^{2} u_{2}^{0}}{\partial x_{2}^{2}}\left(x_{1}, 0\right)\left[\bar{\gamma}\left(1-x_{2}\right)+\frac{\epsilon \bar{\gamma} \bar{\beta}}{1+\epsilon \bar{\beta}}\left(1-x_{2}\right)\right]=: u^{2} .
\end{aligned}
$$


This means that the averaging process cancels the oscillations providing only macroscopic terms still depending on $\epsilon$. Moreover one has the following compact form of the full boundary layer correctors:

$$
\begin{aligned}
& u_{\epsilon}^{1, \infty}=u^{1}+\epsilon \frac{\partial u^{1}}{\partial x_{2}}\left(x_{1}, 0\right)\left(\beta\left(\frac{x}{\epsilon}\right)-\bar{\beta}\right), \\
& u_{\epsilon}^{2, \infty}=u^{2}+\epsilon \frac{\partial u^{2}}{\partial x_{2}}\left(x_{1}, 0\right)\left(\beta\left(\frac{x}{\epsilon}\right)-\bar{\beta}\right)+\frac{\epsilon^{2}}{2} \frac{\partial^{2} u^{2}}{\partial x_{2}^{2}}\left(x_{1}, 0\right)\left(\gamma\left(\frac{x}{\epsilon}\right)-\bar{\gamma}\right) .
\end{aligned}
$$

At this point, if one computes the boundary value problem that $u^{1}$ and $u^{2}$ solve in the smooth domain, one obtains Robin and Wentzel type problems. Namely, $u^{1}$ solves:

$$
\left\{\begin{array}{l}
-\Delta u^{1}=C, \quad \forall x \in \Omega^{0}, \\
u^{1}=\epsilon \bar{\beta} \frac{\partial u^{1}}{\partial x_{2}}, \quad \forall x \in \Gamma^{0}, \quad u^{1}=0, \quad \forall x \in \Gamma^{1}, \\
u^{1} \text { is } x_{1} \text {-periodic on } \Gamma_{\text {in }} \cup \Gamma_{\text {out }},
\end{array}\right.
$$

whose explicit solution reads:

$$
u^{1}(x)=-\frac{C}{2}\left(x_{2}^{2}-\frac{x_{2}}{1+\epsilon \bar{\beta}}-\frac{\epsilon \bar{\beta}}{1+\epsilon \bar{\beta}}\right)
$$

while the second order wall-law $u^{2}$ satisfies the following boundary value problem:

$$
\left\{\begin{array}{l}
-\Delta u^{2}=C, \quad \forall x \in \Omega^{0}, \\
u^{2}=\epsilon \bar{\beta} \frac{\partial u^{2}}{\partial x_{2}}+\frac{\epsilon^{2}}{2} \bar{\gamma} \frac{\partial^{2} u^{2}}{\partial x_{2}^{2}}, \quad \forall x \in \Gamma^{0}, \\
u^{2}=0, \quad \forall x \in \Gamma^{1}, u^{2} \text { is } x_{1} \text {-periodic on } \Gamma_{\text {in }} \cup \Gamma_{\text {out }} .
\end{array}\right.
$$

4.2. Existence and uniqueness of the second order wall-law. The boundary conditions of problem (13) contain second order normal derivatives (in the literature, these are called conditions of Wentzel type [23, 6, 14]). In this framework existence and uniqueness are not so standard: we provide them here for the sake of being self-contained. First we transform the second order normal boundary term in a tangential term of the same order. Then using the appropriate test function space, we can apply Green's formula on tangential directions and symmetrise the bilinear form associated to the problem.

Lemma 4.1. Under Hypotheses 2.1, the system (13) admits a unique solution in $H_{\#}^{1,1}\left(\Omega^{0}\right)$ $=\left\{v \in H_{\Gamma^{1}}^{1}\left(\Omega^{0}\right) ; v \in H^{1}\left(\Gamma^{0}\right)\right\}$, where $H_{\Gamma^{1}}^{1}$ is the set of functions belonging to $H^{1}\left(\Omega^{0}\right)$, $x_{1}$-periodic on $\Gamma_{\text {in }} \cup \Gamma_{\text {out }}$ and vanishing on $\Gamma^{1}$.

Proof. The boundary condition shall be transformed thanks to the first equation of (13) into

$$
u=\epsilon \bar{\beta} \frac{\partial u}{\partial x_{2}}+\frac{\epsilon^{2}}{2} \bar{\gamma} \frac{\partial^{2} u}{\partial x_{2}^{2}}=\epsilon \bar{\beta} \frac{\partial u}{\partial x_{2}}+\frac{\epsilon^{2}}{2} \bar{\gamma}\left(-C-\frac{\partial^{2} u}{\partial x_{1}^{2}}\right), \quad \forall x \in \Gamma^{0}
$$


Because $P^{0}$ does not intersect $\Gamma$, and thanks to the maximum principle, $\beta>0$ a.e. in $Z^{+} \cup P$. This implies that $\bar{\beta}>0$, which allows the weak formulation [14]:

$$
\begin{aligned}
\frac{1}{\epsilon \bar{\beta}}(u, v)_{\Gamma^{0}} & +(\nabla u, \nabla v)_{\Omega^{0}}-\epsilon \frac{\bar{\gamma}}{2 \bar{\beta}}\left[\left(\frac{\partial u}{\partial x_{1}} v\right)\left(x_{1}, 0\right)\right]_{x_{1}=0}^{x_{1}=L}-\epsilon \frac{\bar{\gamma}}{2 \bar{\beta}}\left(\frac{\partial u}{\partial x_{1}} \frac{\partial v}{\partial x_{1}}\right)_{\Gamma^{0}} \\
& =(C, v)_{\Omega^{0}}-\epsilon \frac{\bar{\gamma}}{2 \bar{\beta}}(C, v)_{\Gamma^{0}},
\end{aligned}
$$

where the third term of the left-hand side vanishes thanks to the periodicity of the solution and of the corresponding test functions of $H_{\#}^{1}\left(\Omega^{0}\right)$. We have obtained a symmetric problem. Because $\bar{\gamma} \in[-1,0[$ and $\bar{\beta} \in] 0,1]$, setting

$$
\begin{aligned}
a(u, v) & =\frac{1}{\epsilon \bar{\beta}}(u, v)_{\Gamma^{0}}+(\nabla u, \nabla v)_{\Omega^{0}}-\epsilon \frac{\bar{\gamma}}{2 \bar{\beta}}\left(\frac{\partial u}{\partial x_{1}} \frac{\partial v}{\partial x_{1}}\right)_{\Gamma^{0}}, v \in H_{\#}^{1,1}\left(\Omega^{0}\right), \\
l(v) & =(C, v)_{\Omega}-\epsilon \frac{\bar{\gamma}}{2 \bar{\beta}}(C, v)_{\Gamma^{0}}
\end{aligned}
$$

one obtains a variational formulation where $a$ is coercive, $H_{\#}^{1,1}\left(\Omega^{0}\right)$ being endowed with the norm

$$
\|u\|_{H_{\#}^{1,1}\left(\Omega^{0}\right)}=\|u\|_{H^{1}\left(\Omega^{0}\right)}+\|u\|_{H^{1}\left(\Gamma^{0}\right)} .
$$

Moreover, $a$ and $l$ are continuous on $H_{\#}^{1,1}\left(\Omega^{0}\right)$; thus the problem is solvable by the LaxMilgram theorem. By the way, we derive the following energy estimates, which describe the dependence of various norms upon $\epsilon$ :

$$
\|u\|_{L^{2}\left(\Gamma^{0}\right)} \leq \sqrt{\epsilon} C, \quad\left\|\frac{\partial u}{\partial x_{1}}\right\|_{L^{2}\left(\Gamma^{0}\right)} \leq \frac{C}{\sqrt{\epsilon}} .
$$

Note that when $\epsilon$ goes to zero, our approximation leaves $H_{\#}^{1,1}\left(\Omega^{0}\right)$ moving to $H_{\Gamma^{1} \cup \Gamma^{0}}^{1}\left(\Omega^{0}\right)$ : we lose control over the tangential derivative on the boundary.

In the particular case of a straight domain $\Omega^{0}$ this unique solution is explicit and reads

$$
u^{2}(x)=-\frac{C}{2}\left(x_{2}^{2}-\frac{x_{2}\left(1+\epsilon^{2} \bar{\gamma}\right)}{1+\epsilon \bar{\beta}}-\frac{\epsilon(\bar{\beta}-\epsilon \bar{\gamma})}{1+\epsilon \bar{\beta}}\right) .
$$

4.3. Macroscopic error estimate. When replacing the Poiseuille profile in $\Omega^{0}$ by $u^{1}$ or $u^{2}$, one can compute the corresponding error estimates.

Proposition 5. Let $u^{\epsilon}$ be the solution of (2) and $u^{1}$ (resp. $u^{2}$ ) be the solution of (11) (resp. (13) ). Under Hypotheses 2.1 one has

$$
\left\|u^{\epsilon}-u^{1}\right\|_{L^{2}\left(\Omega^{0}\right)} \leq C \epsilon^{\frac{3}{2}} \text { and }\left\|u^{\epsilon}-u^{2}\right\|_{L^{2}\left(\Omega^{0}\right)} \leq C \epsilon^{\frac{3}{2}} .
$$

Proof. We only compute the error of the second order approximation, the case of $u^{1}$ being identical. We take advantage of the estimates obtained in Proposition 2 by inserting the full boundary layer corrector $u_{\epsilon}^{2, \infty}$ between $u^{\epsilon}$ and $u^{2}$ :

$$
\begin{aligned}
u^{\epsilon}-u^{2} & =u^{\epsilon}-u_{\epsilon}^{2, \infty}+u_{\epsilon}^{2, \infty}-u^{2} \\
& =u^{\epsilon}-u_{\epsilon}^{2, \infty}+\epsilon \frac{\partial u^{2}}{\partial x_{2}}\left(x_{1}, 0\right)\left(\beta\left(\frac{x}{\epsilon}\right)-\bar{\beta}\right)+\frac{\epsilon^{2}}{2} \frac{\partial^{2} u^{2}}{\partial x_{2}^{2}}\left(x_{1}, 0\right)\left(\gamma\left(\frac{x}{\epsilon}\right)-\bar{\gamma}\right),
\end{aligned}
$$


where we used the compact form exhibited in (10). Then, one gets

$$
\begin{aligned}
\left\|u^{\epsilon}-u^{2}\right\|_{L^{2}\left(\Omega^{0}\right)} & \leq\left\|u^{\epsilon}-u_{\epsilon}^{2, \infty}\right\|_{L^{2}\left(\Omega^{0}\right)} \\
& +K \epsilon\left(\left(1+\epsilon^{2}\right)\left\|\beta\left(\frac{\cdot}{\epsilon}\right)-\bar{\beta}\right\|_{L^{2}\left(\Omega^{0}\right)}+\epsilon\left\|\gamma\left(\frac{\cdot}{\epsilon}\right)-\bar{\gamma}\right\|_{L^{2}\left(\Omega^{0}\right)}\right) .
\end{aligned}
$$

Thanks to Proposition 2 and the last estimate in the claim of Theorem A.1. one gets the desired result.

REMARK 4.1. This result is crucial: it shows that the oscillations of the first order boundary layer $\epsilon \partial u^{0} / \partial x_{2}(\beta-\bar{\beta})$ are larger than the second order macroscopic contribution. It is also optimal (see section 6 for numerical evidence). This observation motivates the sections below.

5. Multi-scale wall-laws. In this section we continue the investigation in the sense introduced above. We aim to compute a solution that exists in $\Omega^{0}$ as $u^{1}$ or $u^{2}$ but that performs a better approximation of the exact solution $u^{\epsilon}$ restricted to $\Omega^{0}$. Below we shall show that this concept provides some new multi-scale wall-laws.

5.1. The first order explicit wall-law. How can a first order correction be improved if the non-oscillating second order extension of SAFFMAN-JOSEPH's condition does not help? The answer below will be to take into account some multi-scale features. If we consider the full boundary layer corrector $u_{\epsilon}^{1, \infty}$, it solves (7). Moreover, on the fictitious boundary $\Gamma^{0}$, its value is easily computed, namely

$$
\left.u_{\epsilon}^{1, \infty}\right|_{x_{2}=0}=\left.\left\{u^{1}+\epsilon \frac{\partial u^{1}}{\partial x_{2}}\left(x_{1}, 0\right)\left(\beta\left(\frac{x}{\epsilon}\right)-\bar{\beta}\right)\right\}\right|_{x_{2}=0}=\epsilon \frac{\partial u^{1}}{\partial x_{2}}\left(x_{1}, 0\right) \beta\left(\frac{x_{1}}{\epsilon}, 0\right) .
$$

We use this value as a non-homogeneous Dirichlet boundary condition on $\Gamma^{0}$ for a Poisson problem that is nevertheless homogeneous on $\Gamma^{1}$. Indeed, we consider the following problem:

$$
\left\{\begin{array}{l}
-\Delta \mathcal{U}_{\epsilon}=C, \quad \forall x \in \Omega^{0}, \\
\mathcal{U}_{\epsilon}=\epsilon \frac{\partial u^{1}}{\partial x_{2}}\left(x_{1}, 0\right) \beta\left(\frac{x_{1}}{\epsilon}, 0\right), \quad \forall x \in \Gamma^{0}, \\
\mathcal{U}_{\epsilon}=0, \quad \forall x \in \Gamma^{1}, \quad \mathcal{U}_{\epsilon} \text { is } x_{1} \text {-periodic on } \Gamma_{\text {in }} \cup \Gamma_{\text {out }},
\end{array}\right.
$$

and we claim the following.

Proposition 6. Under Hypotheses 2.1] one gets the following error estimates:

$$
\left\|u^{\epsilon}-\mathcal{U}_{\epsilon}\right\|_{L^{2}\left(\Omega^{0}\right)} \leq c_{12} \epsilon^{\frac{3}{2}}
$$

Proof. Following the same lines as in the proof of Proposition 5 , one inserts the full boundary layer approximation error $r^{1, \infty}:=u^{\epsilon}-u_{\epsilon}^{1, \infty}$ :

$$
r_{\mathrm{bl}}^{1}=u^{\epsilon}-u_{\epsilon}^{1, \infty}+u_{\epsilon}^{1, \infty}-\mathcal{U}_{\epsilon}=r^{1, \infty}-\left[\mathcal{U}_{\epsilon}-u_{\epsilon}^{1, \infty}\right]=: r^{1, \infty}-J .
$$


The first part of the right-hand side has already been estimated (Prop. 2). It remains to estimate the last term $J$ that solves the following system:

$$
\left\{\begin{array}{l}
-\Delta J=0, \quad \forall x \in \Omega^{0}, \\
J=0, \quad \forall x \in \Gamma^{0}, \\
J=\epsilon \frac{\partial u^{1}}{\partial x_{2}}\left(x_{1}, 0\right)\left(\beta\left(\frac{x_{1}}{\epsilon}, \frac{1}{\epsilon}\right)-\bar{\beta}\right), \quad \forall x \in \Gamma^{1}, \\
J \text { is } x_{1} \text {-periodic on } \Gamma_{\text {in }} \cup \Gamma_{\text {out }} .
\end{array}\right.
$$

Using a $y_{2}$-linear lift $s$ that takes away the $\Gamma^{1}$ boundary term (which is exponentially small w.r.t. $\epsilon$ ), and thanks to the Poincaré inequality, we obtain

$$
\|J\|_{L^{2}\left(\Omega^{0}\right)} \leq c_{13}\|J\|_{H^{1}\left(\Omega^{0}\right)} \leq c_{14} e^{-\frac{1}{\epsilon}}
$$

where $c_{13}$ and $c_{14}$ are constants independent of $\epsilon$.

REMARK 5.1. The error in $O\left(\epsilon^{\frac{3}{2}}\right)$ is only due to the first order boundary layer approximation. Indeed the extension of the Poiseuille flow is only linear inside $\Omega^{\epsilon} \backslash \Omega^{0}$. Nevertheless, we avoid errors when neglecting microscopic oscillations in our macroscopic problem as was the case for $u^{1}$ and $u^{2}$.

Remark 5.2. Proposition [ compares $u_{\epsilon}^{1, \infty}$ and $\mathcal{U}_{\epsilon}$. Because they have the same trace on $\Gamma^{0}$, the $L^{2}$ norm of the difference is exponentially small. In the same way, it is not difficult to show that in fact there is a constant $k$ independent of $\epsilon$ s.t.

$$
\left\|u^{\epsilon}-\mathcal{U}_{\epsilon}\right\|_{H^{1}\left(\Omega^{0}\right)}-k e^{-\frac{1}{\epsilon}} \leq\left\|u^{\epsilon}-u_{\epsilon}^{1, \infty}\right\|_{H^{1}\left(\Omega^{0}\right)} \leq\left\|u^{\epsilon}-\mathcal{U}_{\epsilon}\right\|_{H^{1}\left(\Omega^{0}\right)}+k e^{-\frac{1}{\epsilon}}
$$

This will be used in the numerical tests to give an estimate of the error of the first order full boundary layer approximation.

5.2. A second order explicit wall-law. Extending the same ideas as in the subsection above, one sets the following multi-scale problem: find $\mathcal{V}_{\epsilon} \in H^{1}\left(\Omega^{0}\right)$ such that

$$
\left\{\begin{array}{l}
-\Delta \mathcal{V}_{\epsilon}=C, \quad \forall x \in \Omega^{0}, \\
\mathcal{V}_{\epsilon}=\epsilon \frac{\partial u^{2}}{\partial x_{2}}\left(x_{1}, 0\right) \beta\left(\frac{x_{1}}{\epsilon}, 0\right)+\frac{\epsilon^{2}}{2} \frac{\partial^{2} u^{2}}{\partial x_{2}^{2}} \gamma\left(\frac{x_{1}}{\epsilon}, 0\right), \quad \forall x \in \Gamma^{0}, \\
\mathcal{V}_{\epsilon}=0, \quad \forall x \in \Gamma^{1}, \quad \mathcal{V}_{\epsilon} \text { is } x_{1} \text {-periodic on } \Gamma_{\text {in }} \cup \Gamma_{\text {out }},
\end{array}\right.
$$

for which we can prove, as in Proposition 6 .

Proposition 7. Under Hypotheses 2.1, one gets

$$
\left\|u^{\epsilon}-\mathcal{V}_{\epsilon}\right\|_{L^{2}\left(\Omega^{0}\right)} \leq c_{15} e^{-\frac{1}{\epsilon}}
$$

where the constant $c_{15}$ is independent of $\epsilon$.

5.3. First order implicit wall-laws. Note that the standard averaged wall-laws $u^{1}, u^{2}$ are building blocks of explicit multi-scale approximations $\mathcal{U}_{\epsilon}, \mathcal{V}_{\epsilon}$ solving problems (15), (16). In this part, we look for an implicit approximation that avoids the computation of 
these lower order approximations. Indeed, at first order we propose to solve

$$
\left\{\begin{aligned}
-\Delta \Upsilon_{\epsilon} & =C, \quad \forall x \in \Omega^{0}, \\
\Upsilon_{\epsilon} & =\epsilon \beta\left(\frac{x_{1}}{\epsilon}, 0\right) \frac{\partial \Upsilon_{\epsilon}}{\partial x_{2}}, \quad \forall x \in \Gamma^{0}, \\
\Upsilon_{\epsilon} & =0, \quad \forall x \in \Gamma^{1}, \quad \Upsilon_{\epsilon} \text { is } x_{1} \text {-periodic on } \Gamma_{\text {in }} \cup \Gamma_{\text {out }} .
\end{aligned}\right.
$$

We give here a first result of this kind.

THEOREM 5.1. Under Hypotheses 2.1 there exists a unique solution $\Upsilon_{\epsilon} \in H_{\Gamma^{1}}^{1}\left(\Omega^{0}\right)$ of problem (17). Moreover, one gets

$$
\left\|u^{\epsilon}-\Upsilon_{\epsilon}\right\|_{L^{2}\left(\Omega^{0}\right)} \leq c_{16} \epsilon^{\frac{3}{2}}
$$

where $c_{16}$ is a constant independent of $\epsilon$.

Proof. There exists a unique solution $\Upsilon_{\epsilon}$ solving (17). Indeed, the weak formulation of (17) reads:

$$
\begin{aligned}
a(u, v) & :=(\nabla u, \nabla v)_{\Omega^{0}}+\left(\frac{\partial u}{\partial x_{2}}, v\right)_{\Gamma^{0}} \\
& =(\nabla u, \nabla v)_{\Omega^{0}}+\left(\frac{u}{\epsilon \beta}, v\right)_{\Gamma^{0}}=(C, v)_{\Omega^{0}}=: l(v), \quad \forall v \in H_{\Gamma^{1}}^{1}\left(\Omega^{0}\right) .
\end{aligned}
$$

At the microscopic level, we suppose that $P^{0}$ does not cross $\Gamma$; thus there exists a minimal distance $\delta>0$ separating them. By the maximum principle, $\beta$ is bounded: $\beta \in[\delta ; 1]$. Thus $1 / \beta$ is bounded a.e. The bilinear form $a$ is continuous coercive in $H_{\Gamma^{1}}^{1}\left(\Omega^{0}\right)$, the linear form $l$ is continuous as well, and thus existence and uniqueness follow by the LaxMilgram theorem. To estimate this new approximation's convergence rate we add and substract $\mathcal{U}_{\epsilon}$, the explicit wall-law between $u^{\epsilon}$ and $\Upsilon_{\epsilon}$ :

$$
r_{\mathrm{bl}, \mathrm{i}}^{1}:=u^{\epsilon}-\Upsilon_{\epsilon}=u^{\epsilon}-\mathcal{U}_{\epsilon}+\mathcal{U}_{\epsilon}-\Upsilon_{\epsilon}=r_{\mathrm{bl}}^{1}+\mathcal{U}_{\epsilon}-\Upsilon_{\epsilon}=: r_{\mathrm{bl}}^{1}+\Theta
$$

$\Theta$ is the solution of the boundary value problem reading:

$$
\left\{\begin{aligned}
-\Delta \Theta & =0, \quad \forall x \in \Omega, \\
\Theta & =\epsilon \beta\left[\frac{\partial u^{1}}{\partial x_{2}}-\frac{\partial \Upsilon_{\epsilon}}{\partial x_{2}}\right], \quad \forall x \in \Gamma^{0}, \\
\Theta & =0, \quad \forall x \in \Gamma^{1}, \quad \Theta \text { is } x_{1} \text {-periodic on } \Gamma_{\text {in }} \cup \Gamma_{\text {out }} .
\end{aligned}\right.
$$

We re-express the boundary condition on $\Gamma^{0}$, introducing a Robin like condition, namely:

$$
\Theta-\epsilon \beta \frac{\partial \Theta}{\partial x_{2}}=\epsilon \beta\left[\frac{\partial u^{1}}{\partial x_{2}}-\frac{\partial \mathcal{U}_{\epsilon}}{\partial x_{2}}\right], \quad \forall x \in \Gamma^{0},
$$

where the right-hand side is explicitly known. We have the following weak formulation:

$$
-(\Delta \Theta, v)_{\Omega^{0}}=-\left(\frac{\partial \Theta}{\partial \mathbf{n}}, v\right)_{\Gamma^{0}}+(\nabla \Theta, \nabla v)_{\Omega^{0}}=0, \quad \forall v \in H_{\Gamma^{1}}^{1}\left(\Omega^{0}\right),
$$

where the space $H_{\Gamma^{1}}^{1}\left(\Omega^{0}\right)$ contains $H^{1}\left(\Omega^{0}\right)$ functions vanishing on $\Gamma^{1}$. Then using (19) one writes

$$
a(\Theta, v)=(\nabla \Theta, \nabla v)_{\Omega^{0}}+\left(\frac{\Theta}{\epsilon \beta}, v\right)_{\Gamma^{0}}=\left(\frac{\partial u^{1}}{\partial x_{2}}-\frac{\partial \mathcal{U}_{\epsilon}}{\partial x_{2}}, v\right)_{\Gamma^{0}}
$$


We remark that the right-hand side is in fact a boundary term of another comparison problem and we set $z=u^{1}-\mathcal{U}_{\epsilon}$, where $z$ is harmonic and solves

$$
\left(\frac{\partial z}{\partial x_{2}}, v\right)_{\Gamma^{0}}=-(\Delta z, v)_{\Omega^{0}}-(\nabla z, \nabla v)_{\Omega^{0}}, \quad \forall v \in H_{\Gamma^{1}}^{1}\left(\Omega^{0}\right) .
$$

Estimates of the gradient. We have recovered a simpler problem that reads

$$
a(\Theta, v)=-(\nabla z, \nabla v)_{\Omega^{0}}, \quad \forall v \in H_{\Gamma_{1}}^{1}\left(\Omega^{0}\right) .
$$

Thanks to Proposition 2 and Proposition [ 6 one gets

$$
\|\nabla \Theta\|_{L^{2}\left(\Omega^{0}\right)} \leq\|\nabla z\|_{L^{2}\left(\Omega^{0}\right)} \leq\left\|\nabla\left(u^{\epsilon}-u^{1}\right)\right\|_{L^{2}\left(\Omega^{\epsilon}\right)}+\left\|\nabla\left(u^{\epsilon}-\mathcal{U}_{\epsilon}\right)\right\|_{L^{2}\left(\Omega^{0}\right)} \leq 2 c_{17} \epsilon,
$$

where $K$ is a constant independent of $\epsilon$.

Estimate of the trace. The control on the interior term enables us to recover trace estimates

$$
\|\Theta\|_{L^{2}\left(\Gamma^{0}\right)}^{2} \leq\|\beta\|_{L^{\infty}(\Gamma)} \int_{0}^{L} \frac{\Theta^{2}\left(x_{1}, 0\right)}{\beta\left(\frac{x_{1}}{\epsilon}, 0\right)} d x_{1} \leq \epsilon\|\nabla \Theta\|_{L^{2}\left(\Omega^{0}\right)}\|\nabla z\|_{L^{2}\left(\Omega^{0}\right)} \leq c_{17}^{2} \epsilon^{3} .
$$

Final estimate. By the dual problem, and the trace estimates above, we finally obtain

$$
\|\Theta\|_{L^{2}\left(\Omega^{0}\right)} \leq c_{18}\|\Theta\|_{L^{2}\left(\Gamma^{0}\right)} \leq c_{19} \epsilon^{\frac{3}{2}},
$$

Recalling relation (18), one gets

$$
\left\|r_{\mathrm{bl}, \mathrm{i}}^{1}\right\|_{L^{2}\left(\Omega^{0}\right)} \leq\left\|r_{\mathrm{b} 1}^{1}\right\|_{L^{2}\left(\Omega^{0}\right)}+\|\Theta\|_{L^{2}\left(\Omega^{0}\right)},
$$

which ends the proof.

REMARK 5.3. A similar implicit approach could be considered at second order. This should lead one to consider a multi-scale Wentzel condition. It is an open problem to show existence, uniqueness and error estimates as in Theorem 5.1 in this case.

6. Numerical assessment. In order to validate our theoretical results and to show some more interesting features, we propose in this section a set of numerical tests.

We use the finite element method implemented in the well-known freefem++ language [15. The source code of tests presented below is available online 1 In what follows we will compute numerically the approximation error between the exact solution $u^{\epsilon}$ and various wall-laws $\left(u^{1}, u^{2}, \mathcal{U}_{\epsilon}, \mathcal{V}_{\epsilon}, \Upsilon_{\epsilon}\right)$ w.r.t. $\epsilon$. For this sake we introduce in a first step the discretization of the domain $\Omega^{\epsilon}$. Next, we present the detailed procedure to compute either $u^{\epsilon}, \beta, \gamma$ or the wall-laws above.

6.1. Domain discretization. The rough domain that one should discretize is $\Omega^{\epsilon}$; see Fig. 4. The rough boundary is defined as in (1), but here we explicitly set

$$
f\left(y_{1}\right):=-\frac{\left(1+\cos \left(y_{1}\right)\right)}{2}-\delta,
$$

with $\delta$ being a positive constant equal to $5 e-2$. The periodicity of the bottom shape and of the boundary conditions on $\Gamma_{\text {in }} \cup \Gamma_{\text {out }}$ allows us to discretize only a single rough period; i.e., we set

$$
\left.\omega_{r}^{\epsilon}:=\left\{x_{1} \in\right] 0,2 \pi \epsilon\left[\text { and } x_{2} \in\right] \epsilon f\left(x_{1} / \epsilon\right), 0[\}, \quad \omega_{0}^{\epsilon}:=\right] 0,2 \pi \epsilon[\times] 0,1\left[, \quad \omega^{\epsilon}:=\omega_{0}^{\epsilon} \cup \omega_{r}^{\epsilon} .\right.
$$

\footnotetext{
${ }^{1}$ http://ljk.imag.fr/membres/Vuk.Milisic/Software/WallLawTests .edp
} 

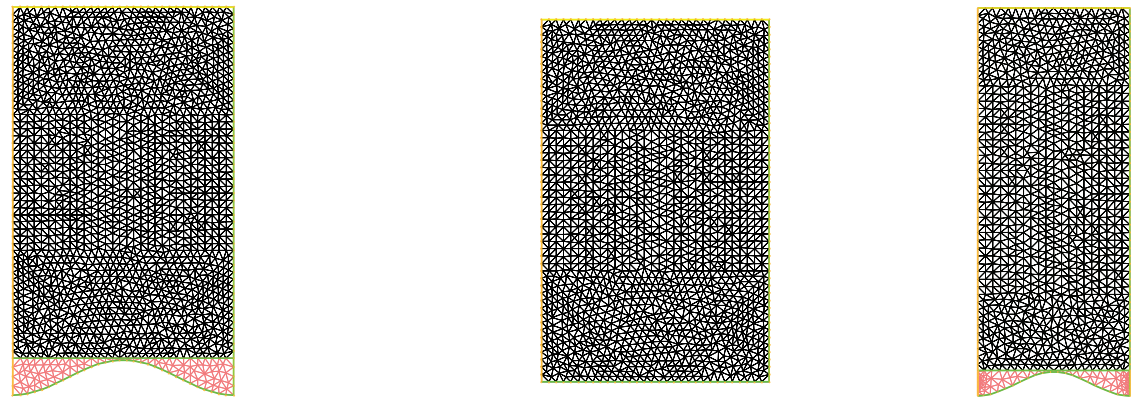

FIG. 5. Meshes $\omega^{\epsilon}, \omega_{r}^{\epsilon}$, and $\left(Z^{+} \cup P\right)_{h}$ when $\epsilon=0.1$ (coarse grids, see below for actual mesh sizes)

The mesh is periodic, i.e., the vertices on $\Gamma_{\text {in }}$ are associated to elements containing edges on $\Gamma_{\text {out }}$ (see p. 142 of the freefem ++ documentation for further information on this facility). For a given $\epsilon$, the meshes of $\omega^{\epsilon}$ and $\omega_{0}^{\epsilon}$ are conforming on the upper part $\left\{x_{2} \geq 0\right\}$. We take several values of $\epsilon$, namely we set $i \in\{1, \ldots, 10\}$ and $\epsilon=q^{i}$, where $q:=0.85$. In order to avoid discretization errors we set $n^{\epsilon}:=90 / \epsilon^{\alpha}, \alpha=0.2$ nodes on the horizontal fictitious boundary, and linearly proportional numbers of nodes on the other boundaries. This gives a mesh size $h$ (maximal diameter of a triangulation, see p.88 [15]) depicted in Fig. [6 as a function of $\epsilon$. This gives that there exists a constant $k$ independent of $\epsilon$ such that

$$
h \leq k \epsilon
$$

which should be used below.

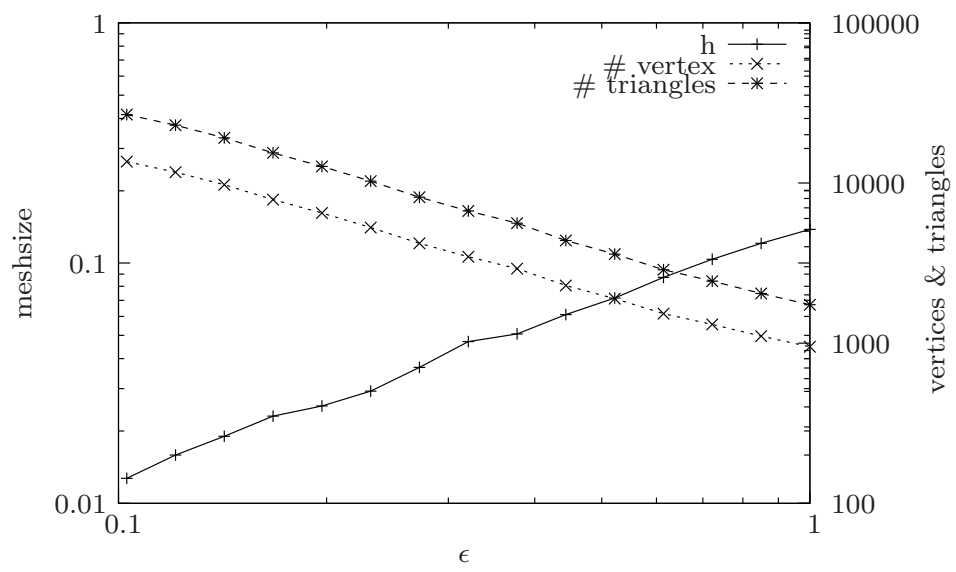

FiG. 6. Mesh size $h$, number of vertices, number of elements 
6.2. The rough solution. For each value of $\epsilon$, we discretize problem (2) using $\mathbb{P}_{2}$ Lagrange finite elements. The matrix obtained after assembling elementary matrices is solved by a direct $L U$ factorization. For the rest of this section, $\mathbb{P}_{2}$ elements and $L U$ factorization are used as well.

6.3. Microscopic cell problems. We compute solutions of cell problems (4) and (8). The domain of definition $Z^{+}$of $\beta$ and $\gamma$ is not bounded. We truncate it by setting $Z_{H}^{+}:=\left\{y \in Z\right.$ s.t. $\left.y_{2}<H\right\}$ and we solve (44) and (8) adding the essential conditions:

$$
\frac{\partial \beta_{H}}{\partial \mathbf{n}}=0, \quad \frac{\partial \gamma_{H}}{\partial \mathbf{n}}=0, \text { on } Z_{H}^{+} .
$$

In [22], the authors show that solutions of these problems are exponentially close to the ones of the problem set on $Z^{+} \cup P$, i.e.,

$$
\left\|\nabla\left(\beta-\beta_{H}\right)\right\|_{L^{2}\left(Z_{H}^{+}\right)} \leq k e^{-H} .
$$

Here we increase $H$ in our discretization of $Z_{H}^{+}$until the variation of the Dirichlet norm of $\beta_{H}, \gamma_{H}$ is lower than $10^{-5}$. Then we compute numerically $\bar{\beta}$ and $\bar{\gamma}$ using a fifth order quadrature formula (see p.116 in [15]). This part is performed once (this step being independent of $\epsilon$ ). For this specific shape $f$, the computations give the following numerical values:

$$
\bar{\beta}=0.432096, \quad \bar{\gamma}=-0.297993 .
$$

Cell problems are computed over a mesh containing 56945 elements and 28911 vertices corresponding to a maximal mesh diameter of 0.126794 .

6.4. Wall-laws. We compute numerically $u_{h}^{1}, u_{h}^{2}, \Upsilon_{\epsilon, h}, \mathcal{U}_{\epsilon, h}, \mathcal{V}_{\epsilon, h}$, solutions of the discrete versions of problems (11), (13), (17), (15), (16). On the fictitious boundary we use the values computed in the cell problems above, either as explicit non-homogenous boundary conditions (as in the variational formulation of problems (15), (16)), or as weights in trace operators (as for problems (11), (13), (17)).
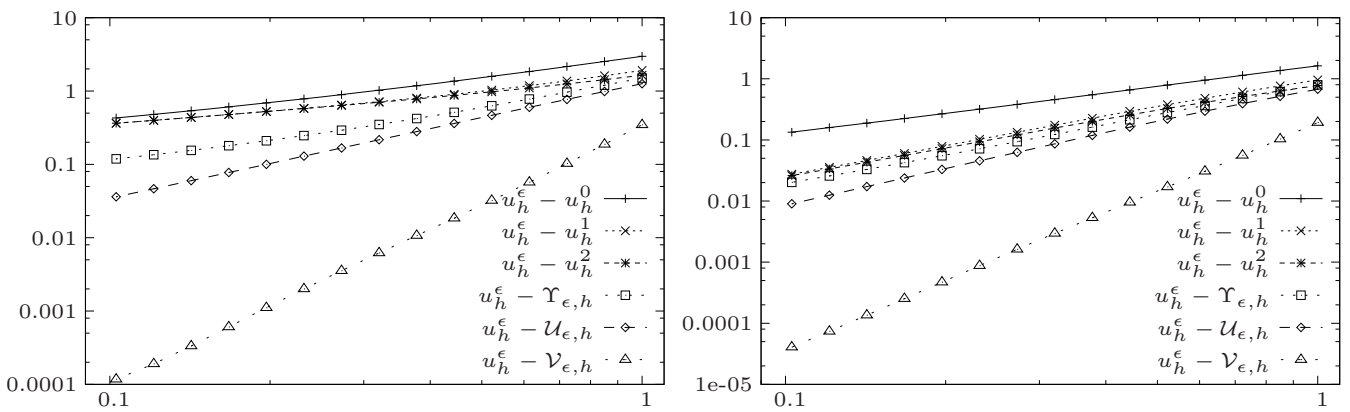

FIG. 7. Computed errors versus $\epsilon$

6.5. Results. We plot in Fig. 7 convergence results w.r.t. the $H^{1}\left(\Omega^{0}\right)$ and $L^{2}\left(\Omega^{0}\right)$ norms: we rescale the errors obtained on $\omega_{0, h}^{\epsilon}$ w.r.t. $\Omega^{0}$. We display in Table 1 the corresponding numerical values for the powers of $\epsilon$ depicted in Fig. 7 . Nodes of the upper part of $\omega^{\epsilon}$ and those of $\omega_{0}^{\epsilon}$ coincide; i.e., there is no interpolation error due to nonconforming meshes. As the problems solved are linear, the orders of convergence seem 


\begin{tabular}{|l||c|c|c|c|c|c|}
\hline$\left\|e_{h}\right\|_{\{\cdot\}}$ & $u_{h}^{\epsilon}-u_{h}^{0}$ & $u_{h}^{\epsilon}-u_{h}^{1}$ & $u_{h}^{\epsilon}-u_{h}^{2}$ & $u_{h}^{\epsilon}-\Upsilon_{\epsilon, h}$ & $u_{h}^{\epsilon}-\mathcal{U}_{\epsilon, h}$ & $u_{h}^{\epsilon}-\mathcal{V}_{\epsilon, h}$ \\
\hline \hline$H^{1}\left(\Omega^{0}\right)$ & 0.9 & 0.7923 & 0.696 & 1.25 & 1.54 & 3.7 \\
\hline$L^{2}\left(\Omega^{0}\right)$ & 1.11 & 1.48 & 1.40 & 1.6245 & 1.776 & 3.77 \\
\hline \multicolumn{6}{c}{ TABLE 1. Numerical orders of convergence for various approxima- } \\
tions: implicit (left) and explicit (right) wall-laws
\end{tabular}

to behave closely to the theoretical predictions. Indeed simulations guarantee theoretical orders of convergence: if one sets $v$ to be one of the wall-law approximations, and $v_{h}$ the corresponding numerical discretisation, one gets

$$
\begin{aligned}
\left|u_{h}^{\epsilon}-v_{h}\right|_{m, \Omega^{0}} & \leq\left|u_{h}^{\epsilon}-u^{\epsilon}\right|_{m, \Omega^{0}}+\left|u^{\epsilon}-v\right|_{m, \Omega^{0}}+\left|u^{\epsilon}-v\right|_{m, \Omega^{0}} \\
& \leq k_{1}\left\{h^{p+1-m}+\epsilon^{\alpha}\right\} \leq k_{2}\left\{\epsilon^{p+1-m}+\epsilon^{\alpha}\right\} \leq k_{3} \epsilon^{\min (p+1-m, \alpha)},
\end{aligned}
$$

where $|\cdot|_{m, \Omega^{0}}$ represents either the $L^{2}\left(\Omega^{0}\right)$ norm $(m=0)$ or the $H^{1}\left(\Omega^{0}\right)$ semi-norm $(m=1)$, and where we used relation (20) and the classical Aubin-Nietsche theorem (see [12, p.52 for instance). This implies that for $p$ great enough (in our case $p=2$ ) only exponential convergence may not be recovered numerically. Results of simulations above show that the implicit wall-laws $u_{h}^{1}, u_{h}^{2}, \Upsilon_{\epsilon, h}$ provide cheaper but less accurate results than explicit wall-laws. This is expected since the latter contain data coming from explicit computations of $u^{1}$ and $u^{2}$ given by formulas (12), (14), whereas the former use only the microscopic data $\bar{\beta}, \bar{\gamma}, \beta(x / \epsilon)$. Nevertheless, among the implicit wall-laws, our implicit multi-scale candidate $\Upsilon_{\epsilon}$ performs the best results thanks to its multi-scale features.

These simulations assess also that the second order wall-law $u^{2}$ does not provide better than first order error estimates. This provides evidence numerically for the optimality of the estimates of Proposition 5 It explains also why one could not distinguish first from second order approximations in [1, p. 209.

Nor in the present work neither in [20, 21] are there theoretical $H^{1}\left(\Omega^{0}\right)$ estimates of $u^{\epsilon}-u^{1}$. We compute this quantity (see Fig. 7 left) numerically. It seems that standard averaged wall-laws do hardly perform better approximations than $\sqrt{\epsilon}$ in $H^{1}\left(\Omega^{0}\right)$. This is rather unexpected, especially when considering that in this norm and for this specific shape, the zeroth order approximation $u_{h}^{0}$ seems better. This is obviously not true in the $L^{2}\left(\Omega^{0}\right)$ norm.

At last, thanks to Remark 5.2. $H^{1}$ estimates of $u_{h}^{\epsilon}-u_{\epsilon}^{1, \infty}$ may be compared with those of $u_{h}^{\epsilon}-\mathcal{U}_{\epsilon, h}$ showing that the results of Propositions 1 and 2 are optimal. We underline that this is due to the presence of the specific right-hand side $C$ in every problem considered above. For instance, setting tests similar to the simple sheared flow (linear $\tilde{u}^{0}$ ) would certainly lead to better convergence rates for first order approximations [3.

\section{Appendix A. The cell problems.}

A.1. Various properties of the first order cell problem's solution. As $Z^{+} \cup P$ is unbounded in the $y_{2}$ direction, we define

$$
D_{0}^{1,2}=\left\{v \in L_{\mathrm{loc}}^{1}\left(Z^{+} \cup P\right) \text { s.t. } \nabla v \in L^{2}\left(Z^{+} \cup P\right)^{2}, v \text { is } y_{1} \text {-periodic, } v=0 \text { on } P^{0}\right\} .
$$


We lift the solution of problem (4) in order to satisfy the homogeneous Dirichlet boundary condition on $P^{0}$ setting $\tilde{\beta}:=\beta+y_{2} \chi_{[P]}$ : it satisfies

$$
\left\{\begin{array}{l}
-\Delta \tilde{\beta}=\delta_{\Gamma}, \text { in } Z^{+} \cup P, \\
\tilde{\beta}=0, \text { on } P^{0}, \\
\tilde{\beta} \text { is } y_{1} \text {-periodic },
\end{array}\right.
$$

where $\delta_{\Gamma}$ is the Dirac function localized on the microscopic fictitious interface $\Gamma$. Then one has the following result.

Theorem A.1. Under Hypotheses 2.1, there exists $\tilde{\beta}$, a unique solution of (44) belonging to $D_{0}^{1,2}$. Moreover, there exists a unique periodic solution $\eta \in H^{\frac{1}{2}}(\Gamma)$ of the following problem:

$$
\langle S \eta, \mu\rangle=\langle 1, \mu\rangle, \quad \forall \mu \in H^{\frac{1}{2}}(\Gamma),
$$

where $\langle$,$\rangle is the H^{-\frac{1}{2}}(\Gamma)-H^{\frac{1}{2}}(\Gamma)$ duality bracket, and $S$ the inverse of the SteklovPoincaré operator (see Appendix A.1). One has the following correspondance between $\beta$ and the interface solution $\eta$ :

$$
\beta=H_{Z^{+}} \eta+H_{P} \eta
$$

where $H_{Z^{+}} \eta$ (resp. $H_{P} \eta$ ) is the $y_{1}$-periodic harmonic extension of $\eta$ on $Z^{+}$(resp. $P$ ). The solution in $Z^{+}$can be written explicitly as a series of Fourier coefficients of $\eta$ and reads:

$$
H_{Z^{+}} \eta=\beta(y)=\sum_{k=-\infty}^{\infty} \eta_{k} e^{i k y_{1}-|k| y_{2}}, \quad \forall y \in Z^{+}, \quad \eta_{k}=\int_{0}^{2 \pi} \eta\left(y_{1}\right) e^{-i k y_{1}} d y_{1} .
$$

In the macroscopic domain $\Omega^{0}$ this leads to

$$
\left\|\beta\left(\frac{\cdot}{\epsilon}\right)-\bar{\beta}\right\|_{L^{2}\left(\Omega^{0}\right)} \leq K \sqrt{\epsilon}\|\eta\|_{H^{\frac{1}{2}}(\Gamma)} .
$$

The existence and uniqueness of solutions of system (4) have been partially proven in [1. The authors consider a truncated domain supplied with a non-local "transparency" condition; the latter is obtained via the Fourier transform. We give here a rigorous proof in the unbounded domain framework.

Proof of Theorem A.1, In what follows we express the cell problem as an inverse Steklov-Poincaré problem solved on the fictitious interface $\Gamma$. This allows us to characterize $\beta$ in the solution of (4) on domains $Z^{+}$and $P$ separately, as depending only on $\eta$, the trace on $\Gamma$. We apply domain decomposition techniques [29]. In a first step we give a simple proof of existence that guarantees the existence of the gradient in $L^{2}\left(Z^{+} \cup P\right)$. The solutions of the cell problems are not in the classical Sobolev spaces because the domain $Z^{+}$is unbounded in the $y_{2}$ direction: the solutions are only locally integrable. For this purpose, we define, for an arbitrary open set $\omega$,

$$
D^{n, p}(\omega)=\left\{v \in L_{\mathrm{loc}}^{1}(\omega) / D^{\alpha} v \in \mathrm{L}^{p}(\omega), \forall \alpha \in \mathbb{Z}^{d^{n}}|\alpha|=n, v \text { is } y_{1} \text {-periodic }\right\} .
$$

In the particular case when $n=1$ and $p=2$, we define $D_{0}^{1,2}(\omega):=\left\{v \in D^{1,2}(\omega) /\left.v\right|_{\partial \omega}=\right.$ $0\}:=V_{0}(\omega)$, which is a Hilbert space for the norm of the gradient.

Lemma A.1. Problem (44) admits a unique solution $\beta$ belonging to $D^{1,2}\left(Z^{+} \cup P\right)$. 
Proof of Lemma A.1. We define the lift $s=y_{2} \chi_{[P]}$ that belongs to $D^{1,2}\left(Z^{+} \cup P\right)$. Setting $\tilde{\beta}=\beta-s$, the lifted problem becomes

$$
\left\{\begin{array}{l}
-\Delta \tilde{\beta}=\delta_{\Gamma}, \quad \text { in } Z^{+} \cup P, \\
\tilde{\beta}=0, \quad \text { on } P^{0},
\end{array}\right.
$$

where $\delta_{\Gamma}$ is the Dirac measure that concentrates on the fictitious interface $\Gamma$. The equivalent variational form of this problem reads

$$
a(\tilde{\beta}, v)=l(v), \quad v \in D_{0}^{1,2}\left(Z^{+} \cup P\right),
$$

where $a(u, v)=(\nabla u, \nabla v)_{Z^{+} \cup P}$ and $l(v)=-(\nabla s, \nabla v)_{P}$. These forms are obviously continuous bilinear (resp. linear) on $D_{0}^{1,2}\left(Z^{+} \cup P\right) \times D_{0}^{1,2}\left(Z^{+} \cup P\right)\left(\right.$ resp. $\left.D_{0}^{1,2}\left(Z^{+} \cup P\right)\right)$. Because of the homogeneous boundary condition the semi-norm of the gradient is a norm. By the Lax-Milgram theorem, the desired result follows.

We define the following spaces:

$$
\begin{aligned}
& V_{1}=D^{1,2}\left(Z^{+}\right), \quad V_{2}=\left\{v \in H^{1}(P) \text { s.t. }\left.v\right|_{P^{0}}=0, \quad v \text { is } y_{1} \text {-periodic }\right\}, \\
& V_{1,0}=\left\{v \in V_{1},\left.\quad v\right|_{\Gamma}=0\right\}, \quad V_{2,0}=\left\{v \in V_{2},\left.\quad v\right|_{\Gamma}=0\right\}, \\
& \Lambda=\left\{\eta \in H^{\frac{1}{2}} \text { s.t. } \eta=\left.v\right|_{\Gamma} \text { for a suitable } v \in D_{0}^{1,2}\left(Z^{+} \cup P\right)\right\} .
\end{aligned}
$$

Lemma A.2. The following domain decomposition problem is equivalent to (22). We look for $\left(\beta_{1}, \beta_{2}\right) \in V_{1} \times V_{2}$ such that

$$
\left\{\begin{array}{l}
a_{1}\left(\beta_{1}, v\right):=\left(\nabla \beta_{1}, \nabla v\right)_{Z^{+}}=0, \quad \forall v \in V_{1,0}, \\
\beta_{1}=\beta_{2}, \quad \text { on } \Gamma, \\
a_{2}\left(\beta_{2}, v\right):=\left(\nabla \beta_{1}, \nabla v\right)_{P}=-(\nabla s, \nabla v)_{P} \equiv 0, \quad \forall v \in V_{2,0}, \\
a_{2}\left(\beta_{2}, \mathcal{R}_{2} \mu\right)=-\left(\nabla s, \nabla \mathcal{R}_{2} \mu\right)-a_{1}\left(\beta_{1}, \mathcal{R}_{1} \mu\right), \quad \forall \mu \in \Lambda,
\end{array}\right.
$$

where $\mathcal{R}_{i}$ denotes any possible extension operator from $\Gamma$ to $V_{i}$.

Proof of Lemma A.2. Let us start by considering the solution $\beta$ of (22). Setting $\beta_{1}=$ $\left.\beta\right|_{Z^{+}}, \beta_{2}=\left.\beta\right|_{P}$, we have that $\beta_{i} \in V_{i}$ and that (23).1, (22). 2 and (23). .3 are trivially satisfied. Moreover, for each $\mu \in \Lambda$, the function $\mathcal{R} \mu$ defined as $\mathcal{R} \mu=\mathcal{R}_{1} \mu \chi_{Z^{+}}+\mathcal{R}_{2} \mu \chi_{P}$ belongs to $V_{0}$. Therefore we have $a(\beta, \mathcal{R} \mu)=(f, \mathcal{R} \mu), \forall \mu \in \Lambda$, which is equivalent to (23). 4 .

On the other hand, let $\beta_{i}$ be the solution of (23). Setting $\beta=\beta_{1} \chi_{\left[Z^{+}\right]}+\beta_{2} \chi_{[P]}$ from (23).2, it follows that $\nabla \beta \in L^{2}\left(Z^{+} \cup P\right)$, and $\left.\beta\right|_{P^{0}}=0$. Then taking $v \in V_{0}$ we set $\mu=v_{\mid \Gamma} \in \Lambda$. Define $\mathcal{R} \mu$ as before; clearly $\left(v_{i}-\mathcal{R}_{i} \mu\right) \in V_{i, 0}$ and from (23).1, (23).3, (23). 4 it follows that

$$
\begin{aligned}
a(\beta, v) & =\sum_{i}\left[a_{i}\left(\beta_{i}, v_{i}-\mathcal{R}_{i} \mu\right)+a_{i}\left(\beta_{i}, \mathcal{R}_{i} \mu\right)\right]=-\left(\nabla s, \nabla \mathcal{R}_{2} \mu\right)_{P} \\
& =-(1, \mu)_{\Gamma}=-(1, v)_{\Gamma}=-(\nabla s, \nabla v)_{P} .
\end{aligned}
$$


The Steklov-Poincaré operator. The Steklov-Poincaré operator $S$ acts between the space of trace functions $\Lambda$ and its dual. More precisely, applying Green's formula and setting $H_{i} \eta$ to be the harmonic lift in $Z^{+}$(resp. $P$ ) for all $\eta \in \Lambda$, we have

$$
\begin{aligned}
\langle S \eta, \mu\rangle & =\sum_{i}\left\langle\frac{\partial}{\partial \nu_{i}} H_{i} \eta, \mu\right\rangle=\int_{Z^{+}} \nabla H_{1} \eta \cdot \nabla \mathcal{R}_{1} \mu+\int_{P} \nabla H_{2} \eta \cdot \nabla \mathcal{R}_{2} \mu \\
& =\sum_{i} a_{i}\left(H_{i} \eta, \mathcal{R}_{i} \mu\right), \quad \forall \eta, \mu \in \Lambda,
\end{aligned}
$$

where $\langle\cdot, \cdot\rangle$ denotes the duality pairing between $\Lambda^{\prime}$ and $\Lambda$. In particular, taking $\mathcal{R}_{i} \mu=$ $H_{i} \mu$, we obtain the following variational representation:

$$
\langle S \eta, \mu\rangle=\sum_{i} a_{i}\left(H_{i} \eta, H_{i} \mu\right), \quad \forall \eta, \mu \in \Lambda .
$$

The linear form on $\Lambda$. We set $l(\mu)$ as follows:

$$
l(\mu)=-\left(\nabla s, \nabla H_{2} \mu\right)_{P}=\left(1, \frac{\partial}{\partial x_{2}} H_{2} \mu\right)_{P}=(1, \mu)_{\Gamma} .
$$

Lemma A.3. The problem:

$$
\text { find } \eta \in \Lambda \text { such that }\langle S \eta, \mu\rangle=l(\mu), \quad \forall \mu \in \Lambda,
$$

admits a unique solution. Moreover this is equivalent to solving (23).

Proof of Lemma A.3. We use the Lax-Milgram framework:

- Continuity:

$$
\langle S \eta, \mu\rangle \leq\|\nabla H \eta\|_{L^{2}\left(Z^{+} \cup P\right)}\|\nabla H \mu\|_{L^{2}\left(Z^{+} \cup P\right)} \leq c_{20}\|\eta\|_{\Lambda}\|\mu\|_{\Lambda},
$$

by well-known estimates for solutions of elliptic boundary value problems [24]. For $H_{1}$ this can be computed explicitly (see below). The continuity of $l$ is obvious.

- Coercivity:

$$
\langle S \eta, \eta\rangle=\|\nabla H \eta\|_{L^{2}\left(Z^{+} \cup P\right)}^{2} \geq c_{21}\left\|H_{2} \eta\right\|_{H^{1}(P)}^{2} \geq c_{22}\|\eta\|_{\Lambda}^{2} .
$$

Then applying the Lax-Milgram theorem one gets the desired result.

To prove the equivalence between (24) and (23), it suffices to separate the harmonic lift $H_{i}$ and the solutions of the Poisson problem with homogeneous boundary conditions and the result follows as in 29, p.10.

$H_{1}$, the harmonic extension in $Z^{+}$. We set $\eta \in \Lambda$. By decomposing in $y_{1}$-Fourier modes, one gets that the solution of

$$
\left\{\begin{array}{l}
\Delta \beta=0, \quad \forall y \in Z^{+}, \\
\beta=\eta, \quad \forall y \in \Gamma,
\end{array}\right.
$$

rewritten as $\beta=\sum_{k} \beta_{k}\left(y_{2}\right) e^{i k y_{1}}, \quad \forall y \in Z^{+}$, should satisfy the following system of ODE's:

$$
\left\{\begin{array}{l}
\beta_{k}^{\prime \prime}-k^{2} \beta_{k}=0, \quad y_{2} \in \mathbb{R}^{+}, \\
\beta_{k}(0)=\eta_{k}, \quad y_{2}=0, \\
\beta_{k}\left(y_{2}\right) \in L^{\infty}\left(\mathbb{R}^{+} ; \mathbb{C}\right),
\end{array}\right.
$$


where $\eta_{k}=\int_{0}^{2 \pi} e^{-i k y_{1}} \eta\left(y_{1}\right) d y_{1}$ are $\eta^{\prime}$ 's Fourier coefficients on $\Gamma$. The solution $\beta_{Z^{+}}$is explicit and reads

$$
H_{1} \eta=\beta_{\mid Z^{+}}=\sum_{k=-\infty}^{\infty} \eta_{k} e^{-|k| y_{2}+i k y_{1}}, \quad \forall y \in Z^{+}
$$

To show exponential convergence towards zero of $\beta-\bar{\beta}$ and $\nabla \beta$ when $y_{2} \rightarrow 0$, we use the same arguments as in the second part of [4, Theorem 2.2.1, p. 637, whose proof is omitted.

Proposition 8. There exists $\alpha_{1} \geq(4 \pi)^{2} / 9$ such that the solution of problem (4) satisfies

$$
\|\beta-\bar{\beta}\|_{L^{2}\left(Z^{+} \cup P, e^{\left.\alpha_{1} y_{2}\right)}\right.} \leq c_{23}\|\nabla \beta\|_{L^{2}\left(Z^{+} \cup P, e^{\left.\alpha_{1} y_{2}\right)}\right.} \leq c_{24},
$$

which implies also $\beta$ 's and $\nabla \beta$ 's exponential decay in the $y_{2}$ direction.

A.2. The second order boundary layer.

Proof of Proposition [3. Problem (8) is equivalent to solving

$$
\left\{\begin{array}{l}
\Delta \tilde{\gamma}=2 \chi_{[P]}, \quad \forall y \in Z^{+} \cup P, \\
\tilde{\gamma}=0, \quad \forall y \in P^{0} .
\end{array}\right.
$$

This, under the previous domain decomposition form, reads: find $\left(\tilde{\gamma}_{Z^{+}}, \tilde{\gamma}_{P}\right)$ such that

$$
\left\{\begin{array}{l}
\left(\nabla \tilde{\gamma}_{Z^{+}}, \nabla v\right)_{Z^{+}}=0, \quad \forall v \in H_{\Gamma}^{1}\left(Z^{+}\right), \\
\tilde{\gamma}_{Z^{+}}=\tilde{\gamma}_{P}, \quad \text { on } \Gamma, \\
\left(\nabla \tilde{\gamma}_{P}, \nabla v\right)_{P}=-(2, v)_{P}, \quad \forall v \in H_{\Gamma \cup P^{0}}^{1}(P), \\
\left(\nabla \tilde{\gamma}_{P}, \nabla \mathcal{R}_{P} \mu\right)_{P}=-\left(2, \mathcal{R}_{P} \mu\right)_{P}-\left(\nabla \tilde{\gamma}_{Z^{+}}, \mathcal{R}_{Z^{+}} \mu\right)_{Z^{+}}, \quad \forall \mu \in H^{\frac{1}{2}}(\Gamma) .
\end{array}\right.
$$

Following the same lines as in the proof above, we write the interface problem:

$$
\begin{gathered}
\langle S \lambda, \mu\rangle=\left(\nabla H_{P} \lambda, \nabla H_{P} \mu\right)+\left(\nabla H_{Z^{+}} \lambda, \nabla H_{Z^{+}} \mu\right), \quad \forall \mu \in H^{\frac{1}{2}}(\Gamma), \\
=-\left(2, H_{P} \mu\right)-\left(\nabla \mathcal{G}_{2}, \nabla H_{P} \mu\right)=: l(\mu), \quad \forall \mu \in H^{\frac{1}{2}}(\Gamma),
\end{gathered}
$$

where $\mathcal{G}_{2}$ is the solution of the homogeneous Poisson problem:

$$
\left\{\begin{array}{l}
\Delta \mathcal{G}_{2}=2, \quad \forall y \in P, \\
\mathcal{G}_{2}=0, \quad \forall y \in P^{0} \cup \Gamma, \\
\mathcal{G}_{2} \quad \text { is } y_{1} \text {-periodic. }
\end{array}\right.
$$

One gets the continuity of the linear form again, thanks to the properties of the harmonic lifts [24, 11]:

$$
|l(\mu)|=\left|-\left(2, H_{P} \mu\right)-\left(\nabla \mathcal{G}_{2}, \nabla H_{P} \mu\right)\right| \leq c_{25}\left\|H_{P} \mu\right\|_{H^{1}(P)} \leq c_{26}\|\mu\|_{H^{\frac{1}{2}}(\Gamma)} .
$$

Again, by the Lax-Milgram theorem, one gets the desired result. 


\section{REFERENCES}

[1] Y. Achdou, O. Pironneau, and F. Valentin, Effective boundary conditions for laminar flows over periodic rough boundaries., J. Comput. Phys. 147 (1998), no. 1, 187-218 (English). MR1657773 (99j:76086)

[2] Y. Amirat, G. A. Chechkin, and R. R. Gadyl'shin, Asymptotics of simple eigenvalues and eigenfunctions for the Laplace operator in a domain with oscillating boundary, Zh. Vychisl. Mat. Mat. Fiz. 46 (2006), no. 1, 102-115. MR2239730 (2007d:35009)

[3] Y. Amirat and J. Simon, Influence of rugosity in laminar hydrodynamics, C.R. Acad. Sci. Paris 323 (1996), no. I, 313-318. MR 1404780 (97f:76025)

[4] I. Babuška, Solution of interface problems by homogenization. parts I and II, SIAM J. Math. Anal. 7 (1976), no. 5, 603-645. MR0509273 (58:23013a)

[5] A. Basson and D. Gérard-Varet, Wall laws for fluid flows at a boundary with random roughness, preprint.

[6] M. Bourlard, A. Maghnouji, S. Nicaise, and L. Paquet, Asymptotic expansion of the solution of a mixed Dirichlet-Ventcel problem with a small parameter, Asymptot. Anal. 28 (2001), no. 3-4, 241-278. MR 1878796 (2002k:35068)

[7] D. Bresch and V. Milisic, Towards implicit multi-scale wall-laws, C. R. Math. Acad. Sciences Paris 346 (2008), 833-838. MR2441916

[8] V. Busuioc and D. Iftimie, A non-Newtonian fluid with Navier boundary conditions, J. Dynamics and Diff. Eqs. 18 (2006), no. 4, 1130-1141 (English). MR2229981(2007c:35134)

[9] Th. Clopeau, A. Mikelić, and R. Robert, On the vanishing viscosity limit for the $2 D$ incompressible Navier-Stokes equations with the friction type boundary conditions, Nonlinearity 11 (1998), no. 12, 179-200 (English). MR 1660366 (99g:35102)

[10] S. Colin, P. Lalond, and C. Caen, Validation of a second order slip flow model in rectangular microchannels, Heat Transfer Engineering 25 (2004), no. 3, 23-30 (English).

[11] A. Ern and J.-L. Guermond, Theory and practice of finite elements, Applied Mathematical Series, vol. 159, Springer-Verlag, New York, 2004. MR2050138 (2005d:65002)

[12] V. Girault, Approximations variationnelles des e.d.p., Lecture notes, Master Degree, 2005-2006.

[13] Antoine Gloria, An analytical framework for the numerical homogenization of monotone elliptic operators and quasiconvex energies, Multiscale Model. Simul. 5 (2006), no. 3, 996-1043 (electronic). MR 2272308

[14] D. Gómez, M. Lobo, S. A. Nazarov, and E. Pérez, Spectral stiff problems in domains surrounded by thin bands: Asymptotic and uniform estimates for eigenvalues, J. Math. Pures Appl. (9) 85 (2006), no. 4, 598-632. MR2216309

[15] F. Hecht, O. Pironneau, A. Le Hyaric, and K. Ohtsuka, Freefem ++ (version 2.24-3), Laboratoire Jacques-Louis Lions, Université Pierre et Marie Curie, 2008, http://www.freefem.org/ff++/ $\mathrm{ftp} / \mathrm{freefem++doc.pdf.}$

[16] D. Iftimie and G. Planas, Inviscid limits for the Navier-Stokes equations with Navier friction boundary conditions., Nonlinearity 19 (2006), no. 3, 899-918 (English). MR2214949(2007c:35130)

[17] D. Iftimie, G. Raugel, and G. Sell, Navier-Stokes equations in thin 3D domains with Navier boundary condition, Indiana Univ. Math. J. 156 (2007), 1083-1156. MR 2333468

[18] D. Iftimie and F. Sueur, Viscous boundary layers for the Navier-Stokes equations with the Navier slip conditions, In preparation.

[19] W. Jäger and A. Mikelić, On the interface boundary condition of Beavers, Joseph, and Saffman., SIAM J. Appl. Math. 60 (2000), no. 4, 1111-1127 (English). MR1760028 (2001e:76122)

[20] _ On the roughness-induced effective boundary conditions for an incompressible viscous flow, J. Diff. Equations 170 (2001), 96-122. MR1813101|(2002b:76049)

[21] _ Couette flows over a rough boundary and drag reduction., Commun. Math. Phys. 232 (2003), no. 3, 429-455 (English). MR1952473 (2003j:76025)

[22] W. Jäger, A. Mikelić, and N. Neuss, Asymptotic analysis of the laminar viscous flow over a porous bed., SIAM J. Sci. Comput. 22 (2000), no. 6, 2006-2028 (English). MR.1856299 (2002f:76065)

[23] K. Lemrabet, Problème aux limites de Ventcel dans un domaine non régulier, C. R. Acad. Sci. Paris Sér. I Math. 300 (1985), no. 15, 531-534. MR792383 (86e:35037)

[24] J.L. Lions and E. Magenes, Non-homogeneous boundary value problems and applications, Die Grundlehren der mathematischen Wissenschaften, vol. I, Springer-Verlag, 1972 (English). 
[25] M.C. Lopes Filho, H.J. Nussenzveig Lopez, and G. Planas, On the inviscid limits for twodimensional incompressible flow with Navier friction condition, Siam J. Math. Anal. 36 (2006), no. 4, 1130-1141 (English). MR2139203 (2005k:76026)

[26] A.L. Madureira and F. Valentin, Asymptotics of the Poisson problem in domains with curved rough boundaries, SIAM J. Math. Anal. 38 (2006/07), 1450-1473. MR 2286014 (2007j:35031)

[27] J. Nečas, Les méthodes directes en théorie des équations elliptiques, Masson et Cie, Éditeurs, Paris, 1967. MR0227584 (37:3168)

[28] N. Neuss, M. Neuss-Radu, and A. Mikelić, Effective laws for the Poisson equation on domains with curved oscillating boundaries., Applicable Analysis 85 (2006), 479-502. MR2213071(2006k:35012)

[29] A. Quarteroni and A. Valli, Domain decomposition methods for partial differential equations., Numerical Mathematics and Scientific Computation, Oxford Science Publication, Oxford, 1999 (English). MR:1857663(2002i:65002) 